\title{
Shapes and cycles arising at the steady bifurcation with icosahedral symmetry
}

\author{
Rebecca B. Hoyle \\ Department of Mathematics and Statistics, University of Surrey, \\ Guildford, Surrey GU2 7XH, UK.
}

Tel: 01483 682638, Fax: 01483 686071, email: r.hoyle@surrey.ac.uk

September 29, 2003

\begin{abstract}
This paper analyses the steady state bifurcation with icosahedral symmetry. The Equivariant Branching Lemma is used to predict the generic bifurcating solution branches corresponding to each irreducible representation of the icosahedral group $\mathcal{I}_{h}$. The relevant amplitude equations are deduced from the equivariance condition, and used to investigate the stability of bifurcating solutions. It is found that the bifurcation with icosahedral symmetry can lead to competition between twofold, threefold and fivefold symmetric structures, and between solutions with tetrahedral, threefold and twofold symmetry. Stable het-
\end{abstract}


eroclinic cycles between solutions with $\mathcal{D}_{2}^{z}$ symmetry are found to exist in one of the irreps. The theoretical scenarios are compared with the observed behaviour of icosahedral viruses and nanoclusters.

\section{Introduction}

Surprisingly, icosahedral symmetry occurs quite frequently in nature. Many viruses are icosahedral [1]-[7]. Quasicrystals with icosahedral point symmetry were discovered by Shechtman et al. [8] in splatcooled Al-Mn alloy. Shapes with icosahedral symmetry also emerge at the primary steady state bifurcation in systems with $\mathrm{O}(3)$ symmetry [9]-[12]; this has recently been applied to the growth of tumours [13]. The fullerene molecule or "buckyball", a carbon molecule consisting of 60 atoms $\left(\mathrm{C}_{60}\right)$ arranged in a ball with pentagonal and hexagonal faces like a regular football (soccerball), is a truncated icosahedron. Other icosahedral fullerenes made up of larger numbers of carbon atoms also exist. Clusters of metal or rare gas atoms in the gas phase may take on icosahedral symmetry: depending on the number of atoms in the cluster, they are generally in competition with decahedral or face-centred cubic clusters [14]. Such icosahedral nanoclusters may also be found in metal atoms growing on surfaces [14] or in the condensed phase of $\left(\mathrm{C}_{60}\right)$ [15], where the clusters consist of groups of $\left(\mathrm{C}_{60}\right)$ molecules, themselves icosahedral, rather than atoms.

The current work seeks to discover the shapes that emerge at the primary stationary bifurcation in systems with icosahedral symmetry, such as those described above. This will allow us to make predictions about the ways in which icosahedral structures will change shape: for 
example icosahedral clusters will lose their symmetry on gaining or losing an atom. As clusters form, they seek to minimise their potential energy in a dissipative process, and it is reasonable to assume that this evolution can be characterised by an ordinary differential equation. It would be natural to expect that the new cluster configuration would have the symmetry of a solution that emerges as a stable branch at the primary bifurcation. In fact this is only sometimes the case as we shall see in $\S 5$. Similarly it is observed that icosahedral viruses undergo configurational changes as part of their function [2]-[7]. If these occur as the result of some kind of stress, physical compression or stretching being possible examples, then again one might expect symmetry-breaking to occur in such a way that the new shape conforms to the symmetry of a stable solution branch. At present, however, images of icosahedral viruses are typically produced by averaging over many individual images, exploiting the icosahedral symmetry to superimpose pictures taken of different views of the virus [3] so as to capture high resolution details. Thus if the observed configurational changes are icosahedral modes, as appears often to be the case [2] [4][7], it is not possible to tell whether this is in fact due to the averaging used in reconstructing the images. The current paper suggests that structures with less than full icosahedral symmetry may emerge at the primary bifurcation: it might then be possible to use the reduced symmetries to reconstruct the image and investigate whether viruses use the pathways suggested by bifurcation theory in their biological function.

In the following section we set out the irreducible representations of the icosahedral group $\mathcal{I}_{h}$. In section 3 we use the Equivariant Branch- 
ing Lemma to predict the bifurcating solution branches in each case. We use the equivariance condition to deduce the $\mathcal{I}_{h}$-equivariant amplitude equations for each irrep and hence we determine the stability of solution branches. In section 4 we investigate the existence of heteroclinic cycles in the system. The paper concludes with a discussion in section 5 .

\section{The icosahedral group and its irre- ducible representations}

The icosahedral group $\mathcal{I}$ is generated by the elements $g_{2}$ and $g_{3}$, where $\left(g_{2}\right)^{2}=\left(g_{3}\right)^{3}=\left(g_{2} g_{3}\right)^{5}=e$, illustrated in figure 1. It has order 60, and the elements are $g_{5}^{\mu} g_{2 d}^{\sigma}, g_{5}^{\mu} g_{2} g_{5}^{\nu} g_{2 d}^{\sigma}$, for $\mu, \nu=0,1,2,3,4, \sigma=0,1$,

where $g_{2 d} \equiv g_{2} g_{5}^{-1} g_{2} g_{5} g_{2} g_{5}^{-1}, g_{5} \equiv g_{3} g_{2} g_{3}, g_{3} \equiv g_{5}^{2} g_{2} g_{5}^{3} g_{2 d}$ [16]. The icosahedral group is isomorphic to A5, the alternating group of order 5. In the case where we wish to include reflections, the group is $\mathcal{I}_{h}$ and we add an additional generator $g_{c}$, the reflection through the origin $\mathbf{x} \rightarrow-\mathbf{x}$, where $\left(g_{c}\right)^{2}=e$. We will consider the case with reflections in all that follows.

Consider the stationary bifurcation problem

$$
\dot{\mathbf{x}}=\mathbf{f}(\mathbf{x}, \lambda)
$$

in a system with icosahedral symmetry. Here $\mathbf{x}(t)$ represents the state of the system, $t$ is time, the dot denotes the time derivative and $\lambda$ is a bifurcation parameter. The system of equations ( 1 ) is $\mathcal{I}_{h}$-equivariant, and thus

$$
\mathbf{f}(\gamma \cdot \mathbf{x}, \lambda)=\gamma \cdot \mathbf{f}(\mathbf{x}, \lambda), \quad \forall \gamma \in \mathcal{I}_{h}
$$


It is assumed that the zero solution $\mathbf{x}=\mathbf{0}$, which has full icosahedral symmetry, is stationary at the bifurcation point $\lambda=0$, i.e. $\mathbf{f}(\mathbf{0}, 0)=0$, and that at $\lambda=0$ an isolated real eigenvalue (of multiplicity greater than one, in general) passes transversely through zero. The last assumption rules out Hamiltonian systems in particular.

The symmetry of solution branches bifurcating from $\mathbf{x}=\mathbf{0}$ at $\lambda=0$ can be determined from a consideration of the irreducible representations (irreps) and subgroups of $\mathcal{I}_{h}$. There are ten irreps and the character table is given in Table 1 . The subgroups of $\mathcal{I}_{h}$ are given in Table 2 together with their generators and abstract definitions. The lattice of inclusion of these subgroups is shown in figure 2 .

It is of interest to note that the representations of $I_{h}$ based on permutations of the directed lines joining opposite faces, opposite vertices and the midpoints of opposite edges are not irreps. For any given representation, $R$, the number of times, $n_{P}$, that each irrep $P$ appears in $R$ can be calculated according to the formula

$$
n_{P}=\frac{1}{g} \sum_{j} \chi_{R}\left(g_{j}\right) \chi_{P}^{*}\left(g_{j}\right)
$$

where the $g_{j}$ are the group elements, $g$ is the order of the group, $\chi_{R}\left(g_{j}\right)$ is the character of $g_{j}$ in representation $R$ and $\chi_{P}^{*}\left(g_{j}\right)$ is the complex conjugate of its character in irrep $P$ [17]. This formula can be used to deduce that the representation which permutes directed lines joining opposite vertices contains one copy of each of irreps $2-$ and $3-$, the one permuting directed lines joining the centres of opposite faces contains one copy of each of irreps $2-, 3-$ and $4-$, and the one permuting directed lines joining the midpoints of opposite edges contains one copy of each of irreps $2-, 3-, 4-$ and $5-$. 


\section{Bifurcating solution branches and their symmetries}

We shall assume that the system has been reduced to the evolution on the centre manifold, where the dynamics is described by the behaviour of a finite number of modes. A generic steady-state symmetric bifurcation is governed by an absolutely irreducible representation of the symmetry group [18], so we shall consider the action on the centre manifold to be described by one of the irreps of $\mathcal{I}_{h}$. In order to determine the symmetry of the bifurcating solution branches we will use the Equivariant Branching Lemma, which can be stated as follows:

Theorem (The Equivariant Branching Lemma): Let $\Gamma$ be a compact Lie group acting on $\mathbb{R}^{n}$, with Fix $\Gamma=\{0\}$, and let $\dot{\mathbf{x}}=\mathbf{f}(\mathbf{x}, \lambda)$ be a $\Gamma$-equivariant smooth bifurcation problem with $\left.D \mathbf{f}\right|_{(\mathbf{0}, 0)}=0$. Then, for every isotropy $\operatorname{subgroup} \Sigma$ satisfying $\operatorname{dim} \operatorname{Fix}(\Sigma)=1$ there is a unique solution branch $(\mathbf{x}(\lambda), \lambda)$, as long as $\left.D \mathbf{f}_{\lambda}\right|_{(\mathbf{0}, 0)}\left(\mathbf{v}_{\mathbf{0}}\right) \neq 0$ for nonzero $\mathbf{v}_{\mathbf{0}} \in \operatorname{Fix}(\Sigma)$.

This result is stated and proved in [18] pp.83-84.

According to the trace formula ([18] p.76) the axial isotropy subgroups, i.e. those with $\operatorname{dim} \operatorname{Fix}(\Sigma)=1$, must satisfy

$$
\frac{1}{n_{G}} \sum_{i=0}^{n_{G}-1} \chi^{p}\left(g_{i}\right)=1,
$$

where the $g_{i}$ are the elements of the isotropy subgroup, $\chi^{p}\left(g_{i}\right)$ is the character of the element $g_{i}$ under the representation $R^{p}$ of $\Gamma$, and $n_{G}$ is the order of the isotropy subgroup.

The trace formula can now be used to identify the symmetry of the solutions guaranteed by the Equivariant Branching Lemma by 
computing the lefthand side of equation ( 4) for each isotropy subgroup under irrep $R^{p}$ of $\mathcal{I}_{h}$ and finding those for which the calculation gives 1. The results are given in Table 3. For the five-dimensional irreps the table also includes maximal, but non-axial, isotropy subgroups that will be discussed below. Illustrations of solutions with the isotropy subgroups given in Table 3 are presented in figures 3- 6 . In the case where there are no reflections the isotropy subgroups with $\operatorname{dim} \operatorname{Fix}(\Sigma)=1$ are also given in [19]. Irreps $2 \pm, 3 \pm$ and $5 \pm$ lead to competition between structures with twofold, threefold and fivefold symmetry. Irrep $4+$ has tetrahedral competing with threefold symmetry, and irrep 4- leads to tetrahedral/threefold/twofold competition. All of these are highly unusual scenarios.

Amplitude equations

$$
\dot{\mathbf{z}}=\mathbf{g}(\mathbf{z} ; \lambda) \quad \mathbf{z} \in \mathbb{R}^{n_{p}},
$$

governing the dynamics on the centre manifold can be found from the equivariance condition

$$
M_{\gamma} \mathbf{g}(\mathbf{z} ; \lambda)=\mathbf{g}\left(M_{\gamma} \mathbf{z} ; \lambda\right), \quad \forall \gamma \in \mathcal{I}_{h}
$$

where $n_{p}$ is the dimension of the relevant irrep and $M_{\gamma}$ is the matrix representing the group element $\gamma$ in that irrep. Close to onset of the bifurcation the amplitude equations can be expanded in powers of the $z_{j}$, the components of $\mathbf{z}$. The equivariance condition will determine which of these terms can appear on the righthand side of the amplitude equations. Those that are permitted appear with an arbitrary coefficient. Certain features of the amplitude equations may only hold for special values of the coefficients: for example, some solutions may only appear if certain combinations of coefficients are zero. Features 
that hold for general values of the coefficients, in the absence of special constraints, are termed generic. For any particular application the values of the coefficients can be determined from the form of $\mathbf{f}(\mathbf{x}, \lambda)$ for the system in question.

\subsection{One-dimensional irreps}

The only nontrivial one-dimensional irrep is $1-$, which governs solutions with only the rotational symmetries of an icosahedron. They arise at a pitchfork bifurcation, since some of the matrices in the irrep are -1 and hence the equivariance condition becomes $-g(z ; \lambda)=$ $g(-z ; \lambda)$, forbidding terms even in $z$. Thus the amplitude equation is

$$
\dot{z}=\lambda z+c_{3} z^{3}+\ldots
$$

It is easy to show that the stationary solutions are given by $z^{2}=$ $-\lambda / c_{3}$, and perturbations to them have growth rate eigenvalue $-2 \lambda$, so the solutions will be stable if $\lambda>0$ and unstable otherwise.

\subsection{Three-dimensional irreps}

In irreps $2 \pm$ the generators of the group can be represented by the matrices

$$
\begin{aligned}
& M_{g_{5}}= \frac{1}{2}\left(\begin{array}{ccc}
1 / \tau & -\tau & 1 \\
\tau & 1 & 1 / \tau \\
-1 & 1 / \tau & \tau
\end{array}\right), \\
& M_{g_{2}}=\left(\begin{array}{ccc}
-1 & 0 & 0 \\
0 & 1 & 0 \\
0 & 0 & -1
\end{array}\right)
\end{aligned}
$$


where $\tau=(1+\sqrt{5}) / 2$. In irreps $3 \pm$ the corresponding matrices are

$$
\begin{aligned}
M_{g_{5}} & =\frac{1}{2}\left(\begin{array}{ccc}
-\tau & -1 / \tau & 1 \\
1 / \tau & 1 & \tau \\
-1 & \tau & -1 / \tau
\end{array}\right), \\
M_{g_{2}} & =\left(\begin{array}{ccc}
-1 & 0 & 0 \\
0 & 1 & 0 \\
0 & 0 & -1
\end{array}\right) .
\end{aligned}
$$

Both irreps $2+$ and $3+$ have $M_{g_{c}}=I$, while irreps $2-$ and 3 - have $M_{g_{c}}=-I$. Irrep 3- is the natural representation of $\mathcal{I}_{h}$.

The amplitude equations are found from the equivariance condition ( 6). In order to distinguish between solutions with twofold, threefold and fivefold symmetry it is necessary to go to fifth order in the expansion. The calculations are extremely time-consuming and were performed using the computer algebra package Maple, as were the derivations of the amplitude equations for the four- and fivedimensional irreps presented below.

Irreps $2 \pm$ give rise to the amplitude equations

$$
\begin{aligned}
\dot{z}_{1}= & \lambda z_{1}-c_{1} z_{1}\left(z_{1}^{2}+z_{2}^{2}+z_{3}^{2}\right) \\
& +\frac{1}{10}\left(5\left(c_{2}+c_{3}\right)-\sqrt{5}\left(c_{2}-c_{3}\right)\right) z_{1}^{5}+2 c_{3} z_{1}^{3} z_{2}^{2}+2 c_{2} z_{1}^{3} z_{3}^{2}+c_{2} z_{1} z_{2}^{4} \\
& +\left(c_{2}+c_{3}-\sqrt{5}\left(c_{2}-c_{3}\right)\right) z_{1} z_{2}^{2} z_{3}^{2}+c_{3} z_{1} z_{3}^{4}, \\
\dot{z}_{2}= & \lambda z_{2}-c_{1} z_{2}\left(z_{1}^{2}+z_{2}^{2}+z_{3}^{2}\right) \\
& +\frac{1}{10}\left(5\left(c_{2}+c_{3}\right)-\sqrt{5}\left(c_{2}-c_{3}\right)\right) z_{2}^{5}+2 c_{3} z_{2}^{3} z_{3}^{2}+2 c_{2} z_{2}^{3} z_{1}^{2}+c_{2} z_{2} z_{3}^{4} \\
& +\left(c_{2}+c_{3}-\sqrt{5}\left(c_{2}-c_{3}\right)\right) z_{2} z_{3}^{2} z_{1}^{2}+c_{3} z_{2} z_{1}^{4}, \\
\dot{z}_{3}= & \lambda z_{3}-c_{1} z_{3}\left(z_{1}^{2}+z_{2}^{2}+z_{3}^{2}\right) \\
& +\frac{1}{10}\left(5\left(c_{2}+c_{3}\right)-\sqrt{5}\left(c_{2}-c_{3}\right)\right) z_{3}^{5}+2 c_{3} z_{3}^{3} z_{1}^{2}+2 c_{2} z_{3}^{3} z_{2}^{2}+c_{2} z_{3} z_{1}^{4} \\
& +\left(c_{2}+c_{3}-\sqrt{5}\left(c_{2}-c_{3}\right)\right) z_{3} z_{1}^{2} z_{2}^{2}+c_{3} z_{3} z_{2}^{4},
\end{aligned}
$$


where $\mathbf{z}=\left(z_{1}, z_{2}, z_{3}\right)$. (Note that these $c_{i}$ are different from those appearing in the amplitude equations for the 1- irrep above.) The equations are in gradient form such that $\dot{\mathbf{z}}=\nabla V$, where

$$
\begin{aligned}
V= & \frac{1}{2} \lambda\left(z_{1}^{2}+z_{2}^{2}+z_{3}^{2}\right)-\frac{c_{1}}{4}\left(z_{1}^{4}+z_{2}^{4}+z_{3}^{4}\right) \\
& -\frac{c_{1}}{2}\left(z_{1}^{2} z_{2}^{2}+z_{2}^{2} z_{3}^{2}+z_{3}^{2} z_{1}^{2}\right) \\
& +\frac{1}{60}\left(5\left(c_{2}+c_{3}\right)-\sqrt{5}\left(c_{2}-c_{3}\right)\right)\left(z_{1}^{6}+z_{2}^{6}+z_{3}^{6}\right) \\
& +\frac{c_{2}}{2}\left(z_{1}^{4} z_{3}^{2}+z_{2}^{4} z_{1}^{2}+z_{3}^{4} z_{2}^{2}\right)+\frac{c_{3}}{2}\left(z_{1}^{4} z_{2}^{2}+z_{2}^{4} z_{3}^{2}+z_{3}^{4} z_{1}^{2}\right) .
\end{aligned}
$$

For irreps $3 \pm$ the equations are identical apart from a change of sign in front of the $\sqrt{5}$ wherever it appears.

The stationary solutions to these equations are given in Table 4 together with the growth rate eigenvalues for perturbations to the solutions. Closed form representations of the eigenvalues in terms of $\lambda$ and the $c_{i}$ are hard to obtain, but close to onset where $\lambda \ll$ 1 approximations are found easily, and these are given in Table 5. Close to onset the solutions with twofold symmetry $\left(\mathbb{Z}_{2} \times \mathbb{Z}_{2}^{c}\right.$ in irreps $2 / 3+$ and $\mathcal{D}_{2}^{z}$ in irreps $2 / 3-$ ) are always unstable. Fivefold-symmetric solutions $\left(\mathbb{Z}_{5} \times \mathbb{Z}_{2}^{c}\right.$ in irreps $2 / 3+$ and $\mathcal{D}_{5}^{z}$ in irreps $\left.2 / 3-\right)$ are stable for $\lambda>0$ and $c_{2}>c_{3}$ in the case of irreps $2 \pm$, and for $\lambda>0$ and $c_{2}<c_{3}$ in the case of irreps $3 \pm$. Threefold-symmetric solutions $\left(\mathbb{Z}_{3} \times \mathbb{Z}_{2}^{c}\right.$ in irreps $2 / 3+$ and $\mathcal{D}_{3}^{z}$ in irreps 2/3-) are stable for $\lambda>0$ and $c_{2}<c_{3}$ in the case of irreps $2 \pm$, and for $\lambda>0$ and $c_{2}>c_{3}$ in the case of irreps $3 \pm$. So only one type of solution is stable for any given combination of parameter values, and it will have either fivefold or threefold symmetry. 


\subsection{Four-dimensional irreps}

In the four-dimensional irreps $4 \pm$, the generators are represented by the matrices:

$$
\begin{aligned}
& M_{g_{5}}=\left(\begin{array}{llll}
0 & 0 & 0 & -1 \\
1 & 0 & 0 & -1 \\
0 & 1 & 0 & -1 \\
0 & 0 & 1 & -1
\end{array}\right), \\
& M_{g_{2}}=\left(\begin{array}{llll}
0 & 0 & 1 & 0 \\
0 & 0 & 0 & 1 \\
1 & 0 & 0 & 0 \\
0 & 1 & 0 & 0
\end{array}\right) .
\end{aligned}
$$

Irrep 4+ has $M_{g_{c}}=I$, while irrep 4- has $M_{g_{c}}=-I$.

The amplitude equations for irreps $4 \pm$ can be deduced from the equivariance condition and are given in the Appendix. At this order in the expansion, the equations are symmetric under S4, the symmetric group of order 4; at higher order full $\mathcal{I}_{h}$ symmetry will be seen.

The possible solutions at onset for irrep $4+$, together with the eigenvalues governing their stability, can be calculated from the quadratic truncation of the amplitude equation and are given in Table 6 . The only solutions are those predicted using the Equivariant Branching Lemma. None of them are stable at onset, as is to be expected since they bifurcate transcritically [18]. Further from the bifurcation point though, the solutions may gain stability, and the existence of quadratic terms in the amplitude equation gives the possibility of hysteresis between the icosahedral base state and the bifurcating solution branches. In the case $c_{1}=0.5, c_{2}=-1$ and $c_{3}=0.7$ shown in figure 7 there 
is hysteresis between the $\mathcal{T} \times \mathbb{Z}_{2}^{c}$ solutions and the zero solution, and also a region where both the $\mathcal{T} \times \mathbb{Z}_{2}^{c}$ and $\mathcal{D}_{3} \times \mathbb{Z}_{2}^{c}$ solutions are stable.

For the irrep $4-$ the possible solutions at the primary bifurcation are given in Table 7 together with the growth rate eigenvalues for perturbations to the solutions. Again an explicit calculation shows that there are no solutions other than those predicted using the Equivariant Branching Lemma. The $\mathcal{D}_{3}^{z}$ and $\mathcal{D}_{2}^{z}$ solutions can never be stable at onset, whereas the $\mathcal{T}$ (tetrahedral) and $\mathcal{D}_{3}$ solutions can be. The tetrahedral solutions will be stable if $\lambda>0$ and $\left(1+2 c_{3} / c_{2}\right)>0$, while the $\mathcal{D}_{3}$ solutions will be stable if $\lambda>0$ and $\left(c_{2}+2 c_{3}\right)\left(4 c_{2}+5 c_{3}\right)<0$. Both types of solution cannot be stable for any one set of parameter values, since for both to be stable we must have $c_{2}\left(4 c_{2}+5 c_{3}\right)<0$ and if $c_{2}>0$ and $c_{3}<-4 c_{2} / 5$ we must then have $c_{2}+2 c_{3}<-3 c_{2} / 5<0$, whereas the conditions $c_{2}<0$ and $c_{3}>-4 c_{2} / 5$ give $c_{2}+2 c_{3}>-3 c_{2} / 5>0$ : in both cases both types of solution are in fact unstable. However, tetrahedral and $\mathcal{D}_{3}$ solutions can be stable in different parameter regimes, so one might expect to observe structures with these symmetries in practice.

\subsection{Five-dimensional irreps}

The matrices for the five-dimensional irreps $5 \pm$ are as follows:

$$
M_{g 5}=\left(\begin{array}{lllll}
1 & 0 & 0 & 0 & -1 \\
0 & 0 & 0 & 0 & -1 \\
0 & 0 & 0 & 1 & -1 \\
0 & 1 & 0 & 0 & -1 \\
0 & 0 & 1 & 0 & -1
\end{array}\right),
$$




$$
M_{g 2}=\left(\begin{array}{ccccc}
0 & -1 & 1 & 0 & 0 \\
0 & -1 & 0 & 0 & 0 \\
1 & -1 & 0 & 0 & 0 \\
0 & -1 & 0 & 1 & 0 \\
0 & -1 & 0 & 0 & 1
\end{array}\right) .
$$

Irrep 5+ has $M_{g_{c}}=I$, while irrep 5- has $M_{g_{c}}=-I$.

The amplitude equations to cubic order for irreps $5 \pm$ are given in the Appendix.

For irrep $5+$ all the solutions at the primary bifurcation that have nontrivial symmetry are given in Table 8 together with the growth rate eigenvalues for perturbations. The results are calculated using the quadratic truncation of the amplitude equations. It is possible that there are also solutions with trivial symmetry, not guaranteed by the Equivariant Branching Lemma. If they exist at all it is likely that it will only be for certain special values of the parameters. The lattice of isotropy subgroups is shown in Figure 8. For all solutions there are always eigenvalues of both signs, and so no solution is stable at onset. However, at cubic order, analysis of the $\mathcal{D}_{5} \times \mathbb{Z}_{2}^{c}$ and $\mathcal{D}_{3} \times \mathbb{Z}_{2}^{c}$ branches given in the table shows that the solutions can become stable far from onset, as shown in figure 9 for the case $c_{1}=c_{2}=-c_{3}=-c_{4}=-c_{5}=$ 1. Both the $\mathcal{D}_{5} \times \mathbb{Z}_{2}^{c}$ and $\mathcal{D}_{3} \times \mathbb{Z}_{2}^{c}$ solutions can be stable and there are regions where the $\mathcal{D}_{5} \times \mathbb{Z}_{2}^{c}$ solution is stable simultaneously with the zero or $\mathcal{D}_{3} \times \mathbb{Z}_{2}^{c}$ solution, so hysteresis is possible. Since we do not have closed form solutions for the $\mathcal{D}_{2} \times \mathbb{Z}_{2}^{c}$, their behaviour far from onset was not investigated.

The stability of the solution branches at the primary bifurcation in the case of irrep 5- is determined from the cubic truncation of 
the amplitude equations given in the Appendix, and the solutions and their stability are summarised in Table 9. The lattice of isotropy subgroups is shown in Figure 10. The $\mathcal{D}_{3}$ and $\mathcal{D}_{5}$ solutions can be stable at onset if $\lambda>0$ and $\left(11 c_{3}+5 c_{4}+2 c_{5}\right)<0$ for $\mathcal{D}_{3}$ or if $\lambda>0$ and $\left(11 c_{3}+5 c_{4}+2 c_{5} \pm \sqrt{5} c_{3}\right)>0$ for $\mathcal{D}_{5}$. These are mutually exclusive possibilities, and if $0<11 c_{3}+5 c_{4}+2 c_{5}<\sqrt{5}\left|c_{3}\right|$ then neither solution is stable. The $\mathcal{D}_{2}^{z}$ solution is always unstable at onset: if $\left(14 c_{3}+5 c_{4}+2 c_{5}\right)\left(8 c_{3}+5 c_{4}+2 c_{5}\right)>0$ holds the two eigenvalues involving the square root are real and of opposite sign, and if $\left(14 c_{3}+5 c_{4}+2 c_{5}\right)\left(8 c_{3}+5 c_{4}+2 c_{5}\right)<0$ then two of the other eigenvalues have opposite sign. For certain combinations of parameter values there are also submaximal solution branches at the primary bifurcation that are not guaranteed by the Equivariant Branching Lemma. They have symmetry $\mathbb{Z}_{2}^{\text {refl }}$ and are never stable since they have real eigenvalues of opposite sign. It is possible that there are also submaximal primary branches with symmetry $\mathbb{Z}_{2}^{\text {rot }}$ of the types $\left(z_{1}, z_{2}, z_{3}, z_{4}, z_{5}\right)=(v, 0, x, y, y)$ and $\left(z_{1}, z_{2}, z_{3}, z_{4}, z_{5}\right)=(v, w, v, y, y)$. The form of the equations indicates that these would bifurcate from the origin, but closed form solutions proved difficult to determine. Again, if these solutions exist at all it is likely only to be for certain combinations of parameter values. A two-dimensional $\mathcal{D}_{2}$-invariant subspace $(v, 0, v, y, y)$ is found as the intersection of the two $\mathbb{Z}_{2}^{\text {rot }}$ invariant subspaces, and indeed $\mathcal{D}_{2}$ is a maximal isotropy subgroup. There are, however, no stationary solutions to the amplitude equations of the form $(v, 0, v, y, y)$ with $0 \neq v \neq y \neq 0$, and hence no stationary solutions with isotropy subgroup $\mathcal{D}_{2}$. An open possibility is that in some parameter regimes there might be solutions with no equal am- 
plitudes and hence no symmetry. The structures one would expect to observe in practice would be those with $\mathcal{D}_{3}$ or $\mathcal{D}_{5}$ symmetry when the parameter values are such that one of these solutions is stable.

Since viruses often have symmetry $\mathcal{I}$ rather than $\mathcal{I}_{h}$, it is also of some interest to summarise the situation for the bifurcation with symmetry $\mathcal{I}$. The axial and maximal isotropy subgroups are given in Table 10. The relevant amplitude equations are the same as those for the plus irreps in the $\mathcal{I}_{h}$ case, and the solution branches with symmetry group $\Gamma$ at the primary bifurcation will have amplitude $z$ and eigenvalues identical to those for the corresponding branch with isotropy subgroup $\Gamma \times \mathbb{Z}_{2}^{c}$ in the plus irrep of the $\mathcal{I}_{h}$ case.

\section{Heteroclinic cycles}

Heteroclinic cycles connecting type 3' and type 3 solutions of Table 9 are found to exist in the case of the 5- irrep. Before examining the structure of these cycles we will first dismiss the possibility of stable heteroclinic cycles arising at the primary bifurcation in the irreps of dimension less than 5 .

Stable heteroclinic cycles require a saddle-sink connection in the subspace connecting each pair of solutions in the cycle. There are clearly no such cycles in the one-dimensional irrep, nor are there any in the three-dimensional irreps since the amplitude equations are in gradient form for irreps $2 \pm$ and $3 \pm$. In irrep $4-$ neither the solutions with isotropy subgroup $\tau$ nor those with symmetry $\mathcal{D}_{3}$ can be saddles in one subspace and stable nodes (sinks) in another when $\lambda>0$ because in both cases there is one negative eigenvalue $-2 \lambda$ and all 
three remaining eigenvalues have the same sign, so either all are negative, precluding the possibility of a saddle, or the remaining three are positive so that the solutions could only be saddles or unstable nodes (sources) in any subspace. So the only solutions that could form part of a heteroclinic cycle are those with $\mathcal{D}_{3}^{z}$ or $\mathcal{D}_{2}^{z}$ symmetry, types $3 / 3$ ' and $4 / 4^{\prime}$ of Table 7 respectively. Now no trajectories can cross the hyperplanes $z_{i}=0$ or $z_{i}=z_{j}(i \neq j)$, since $\dot{z}_{i}$ and $\dot{z}_{i}-\dot{z}_{j}=0$ respectively are zero there. This means that the only possible connections are between a type 3 and a type 4 solution, a type 3 and a type 4' or a type 4 ' and a type 3 '.

To consider the possible connections between a type 3 solution and types 4 and 4 ', it is helpful to choose a concrete example: if we start with $(x, 0,0,-x)$ (type 3$)$, this can only be connected to $(x, x,-x,-x)$ or $(x,-x, x,-x)$ (both type 4$)$ in the subspace $(z, y,-y, z)$ without crossing $\left(z_{i}=0\right.$ or $z_{i}=z_{j}(i \neq j)$. The type 3 solutions in this space will be sinks if $\left(c_{2}+2 c_{3}\right)<0$ and saddles if $\left(c_{2}+2 c_{3}\right)>0$. Now connecting $(x, 0,0,-x)$ to the type 4 ' solution $(2 x, 2 x, x, 0)$ via the subspace $(z, y+z,(y+z) / 2, y)$ or to $(2 x, x, 2 x, 0)$ via $(z,(y+z) / 2, y+$ $z, y)$ (again the only permitted links), we find that in these subspaces the type 3 solutions will also be sinks if $\left(c_{2}+2 c_{3}\right)<0$ and saddles if $\left(c_{2}+2 c_{3}\right)>0$. Thus the type 3 solutions are of the same stability type in both subspaces in which they could be used as part of a cycle, so the cycle fails at this point. Finally, consider the type 4' solution $(2 x, 2 x, x, 0)$ used to connect to the type 3 solution above: in that subspace the type 4' solutions are sinks if $\left(c_{2}+2 c_{3}\right)>0$ and saddles if $\left(c_{2}+2 c_{3}\right)<0$. The point $(2 x, 2 x, x, 0)$ can also be connected to the type 3 ' solutions $(x, 2 x, x, x)$ and $(2 x, x, x, x)$ via the subspaces 
$(z, y+z,(y+z) / 2, y)$ and $(y+z, z,(y+z) / 2, y)$ respectively. In these subspaces the type 4' solutions are sinks if $\left(c_{2}+2 c_{3}\right)>0$ and saddles if $\left(c_{2}+2 c_{3}\right)<0$, so the type 4' solutions also are of the same stability type in both subspaces and cannot form part of a heteroclinic cycle. There are no further possibilities of forming such cycles, so we conclude that no heteroclinic cycles exist for the 4 - irrep.

Similar arguments to those used for the 4- irrep lead to the conclusion that the only possible connections in the case of the $4+$ irrep are between solution types 1 and 2', 1 and 2, and 1' and 2' of Table 6 . Restricting the evolution to the subspace $(z, y, y, y)$ we find that the solution $(x, 0,0,0)$ (type 1$)$ is a saddle there for $\lambda>0$, while $(0, x, x, x)$ (type $\left.2^{\prime}\right)$ is a sink and $(x, x, x, x)$ (type $\left.1^{\prime}\right)$ is a saddle. Thus type 2' cannot be used in any cycle because it is always a sink when connecting to type $1 / 1^{\prime}$ solutions. In the subspace $(z, 0,0, y)$ the type 1 solution $(x, 0,0,0)$ is a saddle and the type 2 solution $(x, 0,0, x)$ is a sink, thus the type 1 solution is always a saddle when connecting to type $2 / 2$ ' solutions. There cannot then be any heteroclinic cycles in irrep $4+$.

Returning now to the 5 - irrep, it will help to choose a particular example, so we concentrate on the heteroclinic cycle connecting the solutions $(x, 0,-x, 0,0),(0,0,0, x,-x)$ and $(x, 2 x, x, x, x)$, where $x^{2}=$ $-\lambda /\left(10 c_{3}+4 c_{4}+4 c_{5}\right)$ in all cases. The first two points both lie in the subspace $(z, 0,-z, y,-y)$, where the evolution equations take the form

$$
\begin{aligned}
& \dot{y}=\lambda y+2\left(5 c_{3}+2 c_{4}+2 c_{5}\right) y^{3}+\left(-4 c_{3}-c_{4}+2 c_{5}\right) z^{2} y \\
& \dot{z}=\lambda z+2\left(5 c_{3}+2 c_{4}+2 c_{5}\right) z^{3}+\left(2 c_{3}-c_{4}+2 c_{5}\right) y^{2} z
\end{aligned}
$$

The stationary points in this system together with their growth rate 
eigenvalues $e_{1}$ and $e_{2}$ are $\left(z^{2}=-\lambda /\left(10 c_{3}+4 c_{4}+4 c_{5}\right), y=0, e_{1}=-2 \lambda\right.$, $\left.e_{2}=\lambda\left(14 c_{3}+5 c_{4}+2 c_{5}\right) /\left(10 c_{3}+4 c_{4}+4 c_{5}\right)\right),\left(y^{2}=-\lambda /\left(10 c_{3}+4 c_{4}+4 c_{5}\right)\right.$, $\left.z=0, e_{1}=-2 \lambda, e_{2}=\lambda\left(8 c_{3}+5 c_{4}+2 c_{5}\right) /\left(10 c_{3}+4 c_{4}+4 c_{5}\right)\right)$ and $\left(z^{2}=-\lambda\left(8 c_{3}+5 c_{4}+2 c_{5}\right) / \xi, y^{2}=-\lambda\left(14 c_{3}+5 c_{4}+2 c_{5}\right) / \xi, e_{1}=-2 \lambda\right.$, $\left.e_{2}=-2 \lambda\left(8 c_{3}+5 c_{4}+2 c_{5}\right)\left(14 c_{3}+5 c_{4}+2 c_{5}\right) / \xi\right)$ where $\xi$ is defined in Table 9 .

We will consider the case where $\lambda>0$, since we are interested in the possibility of observing this cycle after the bifurcation from the trivial solution. In the parameter regime $5 c_{3}+2 c_{4}+2 c_{5}<0$ and $\left(14 c_{3}+5 c_{4}+2 c_{5}\right)\left(8 c_{3}+5 c_{4}+2 c_{5}\right)<0$ only the pure $z$ or pure $y$ solutions can exist, since for the mixed mode we would have $z^{2} y^{2}=\left(14 c_{3}+\right.$ $\left.5 c_{4}+2 c_{5}\right)\left(8 c_{3}+5 c_{4}+2 c_{5}\right) \lambda^{2} / \xi^{2}<0$, which is obviously forbidden. Now either we have $-\left(5 c_{4}+2 c_{5}\right) / 8<c_{3}<0$ which makes the solution $(z, 0,-z, 0,0)$ a saddle and the solution $(0,0,0, y,-y)$ a sink, or we have $0<c_{3}<-\left(5 c_{4}+2 c_{5}\right) / 8$ which makes $(z, 0,-z, 0,0)$ a sink and $(0,0,0, y,-y)$ a saddle. There are no periodic orbits in the system since any periodic orbit would have to enclose a fixed point, but the coordinate axes on which the fixed points lie are invariant and so no trajectory can cross them. Every trajectory must approach either a fixed-point or a periodic orbit by the Poincaré-Bendixson theorem, so the trajectory leaving the saddle point must approach the sink or the origin or escape to infinity. The origin is an unstable node, so the trajectory will not end there, thus as long as we can show that trajectories come in from infinity, there must be a saddle-sink connection in the subspace.

In polar coordinates $y=r \cos \phi, z=r \sin \phi$ we have

$$
\dot{r}=\lambda r+\frac{3}{2}\left(3 c_{3}+c_{4}+2 c_{5}\right) r^{3}+\frac{1}{2}\left(11 c_{3}+5 c_{4}+2 c_{5}\right) r^{3} \cos ^{2}(2 \phi)
$$


from equations ( 20) and (21). If $11 c_{3}+5 c_{4}+2 c_{5}>0$ then

$$
\dot{r} \leq \lambda r+2\left(5 c_{3}+2 c_{4}+2 c_{5}\right) r^{3},
$$

so since $5 c_{3}+2 c_{4}+2 c_{5}<0$ we have $\dot{r}<0$ for $r^{2}>-\lambda /\left[2\left(5 c_{3}+2 c_{4}+\right.\right.$ $\left.2 c_{5}\right)$ ], and all trajectories enter or stop on the boundary of a circle of radius $\sqrt{-\lambda /\left[2\left(5 c_{3}+2 c_{4}+2 c_{5}\right)\right]}$. If on the other hand $11 c_{3}+5 c_{4}+$ $2 c_{5}<0$ then

$$
\dot{r} \leq \lambda r+\frac{3}{2}\left(3 c_{3}+c_{4}+2 c_{5}\right) r^{3},
$$

and to be sure that all trajectories come in from infinity we must have $3 c_{3}+c_{4}+2 c_{5}<0$, and then all trajectories will enter or stop on the boundary of a circle of radius $\sqrt{-2 \lambda /\left[3\left(3 c_{3}+c_{4}+2 c_{5}\right)\right]}$. In either case trajectories come in from infinity and so there is a saddle-sink connection between $(0,0,0, y,-y)$ and $(z, 0,-z, 0,0)$ in the subspace.

Now the points $(0,0,0, x,-x)$ and $(x, 2 x, x, x, x)$ both lie in the subspace $(y, 2 y, y, y+z, y-z)$, where the equations for $\dot{y}$ and $\dot{z}$ are the same as those given in equations ( 20) and (21) above. We maintain the requirements that $\lambda>0,5 c_{3}+2 c_{4}+2 c_{5}<0$ and $\left(14 c_{3}+\right.$ $\left.5 c_{4}+2 c_{5}\right)\left(8 c_{3}+5 c_{4}+2 c_{5}\right)<0$ hold. The first solution is given by $z^{2}=-\lambda /\left(10 c_{3}+4 c_{4}+4 c_{5}\right), y=0\left(e_{1}=-2 \lambda, e_{2}=\lambda\left(14 c_{3}+5 c_{4}+\right.\right.$ $\left.\left.2 c_{5}\right) /\left(10 c_{3}+4 c_{4}+4 c_{5}\right)\right)$ and the second by $y^{2}=-\lambda /\left(10 c_{3}+4 c_{4}+4 c_{5}\right)$, $z=0\left(e_{1}=-2 \lambda, e_{2}=\lambda\left(8 c_{3}+5 c_{4}+2 c_{5}\right) /\left(10 c_{3}+4 c_{4}+4 c_{5}\right)\right)$. So in this subspace the solution $(0,0,0, x,-x)$ is a sink for the same parameter values at which it is a saddle in the first subspace and vice-versa. Using the same reasoning as before there is a saddle-sink connection in this subspace because the solution $(x, 2 x, x, x, x)$ is a saddle when $(0,0,0, x-x)$ is a sink and vice-versa.

Finally to complete the cycle, consider the subspace $(z+y, 2 z, z-$ $y, z, z)$, which contains the solutions $(x, 2 x, x, x, x)$ and $(x, 0,-x, 0,0)$. 
Again the evolution equations are given by (20) and (21) above. So $(x, 0,-x, 0,0)$ is a saddle when it is a sink in the first subspace (and vice versa), and $(x, 2 x, x, x, x)$ is a saddle when it is a sink in the second subspace and vice versa. Furthermore there is a saddle-sink connection between $(x, 0,-x, 0,0)$ and $(x, 2 x, x, x, x)$.

The heteroclinic cycle is shown diagrammatically in figure 11. This cycle is expected to be stable because each of the stationary points involved has two negative, one positive and two purely imaginary eigenvalues in the full five-dimensional space (see Table 9), so the unstable manifold is one-dimensional and stable manifold two-dimensional. Other similar cycles involving solutions with $\mathcal{D}_{2}^{z}$ symmetry also exist, but there are no stable heteroclinic cycles for $\lambda>0$ involving the $\mathcal{D}_{3}$ solutions because these have either five eigenvalues with negative real part or one with negative and four with positive real part, depending on parameter values, thus ruling out the possibility of finding one subspace of two or more dimensions in which the solution would be a saddle and another subspace in which it would be a sink. Neither are there stable cycles involving the $\mathcal{D}_{5}$ solutions, because these solutions are always of the same stability type in all the three-dimensional subspaces (invariant under $\mathbb{Z}_{2}^{\text {rot }}$ ) in which they appear. This latter reason also rules out any stable cycles involving the $\mathcal{D}_{3} \times \mathbb{Z}_{2}^{c}$ or $\mathcal{D}_{5} \times \mathbb{Z}_{2}^{c}$ solutions in the $5+$ irrep.

Each $\mathcal{D}_{2} \times \mathbb{Z}_{2}^{c}$-invariant subspace in the $5+$ irrep contains three saddle points with $\mathcal{D}_{2} \times \mathbb{Z}_{2}^{c}$ symmetry (the $\mathcal{D}_{2} \times \mathbb{Z}_{2}^{c}$ solutions of Table 8 ) and a node at the origin. The saddles are joined by invariant lines to the origin, and have eigenvalues $-\lambda$ along the invariant line [20] and $3 \lambda$ transverse to it. Since the behaviour in the transverse direction is 
the same for each saddle point, there are no trajectories joining any two of them. Any trajectories leaving the saddle points in the $\mathcal{D}_{2} \times \mathbb{Z}_{2}^{c}$ invariant subspace end up either at the origin, which cannot be part of any cycle as all its eigenvalues are of the same sign, or at infinity. Thus the $\mathcal{D}_{2} \times \mathbb{Z}_{2}^{c}$ solutions cannot form part of a stable heteroclinic cycle.

Finally, there are no stable heteroclinic cycles at the primary bifurcation when we are considering the icosahedral group without reflections, $\mathcal{I}$, because the behaviour in that case is related to that of the plus irreps as explained above.

The heteroclinic cycles in the 5- irrep can exist in parameter regimes where the $\mathcal{D}_{3}$ and $\mathcal{D}_{5}$ solutions bifurcate supercritically and are unstable: for example choose $c_{3}=-1 / 4, c_{4}=1$ and $c_{5}=-1$ for the cycle to run in the direction shown in figure 11 or $c_{3}=3 / 32$, $c_{4}=1 / 5$ and $c_{5}=-1$ for it to run in the opposite direction. Thus we might expect the cycles to be observable in practice.

\section{Conclusion}

In summary, we have found that the possible stable solution branches at onset in a stationary bifurcation with icosahedral $\mathcal{I}_{h}$ symmetry are those with isotropy subgroups $\mathcal{I}, \mathbb{Z}_{2} \times \mathbb{Z}_{2}^{c}, \mathbb{Z}_{3} \times \mathbb{Z}_{2}^{c}, \mathbb{Z}_{5} \times \mathbb{Z}_{2}^{c}, \mathcal{D}_{2}^{z}, \mathcal{D}_{3}^{z}$, $\mathcal{D}_{5}^{z}, \mathcal{T}, \mathcal{D}_{3}$ or $\mathcal{D}_{5}$. In addition $\mathcal{T} \times \mathbb{Z}_{2}^{c}, \mathcal{D}_{3} \times \mathbb{Z}_{2}^{c}$ and $\mathcal{D}_{5} \times \mathbb{Z}_{2}^{c}$ solutions can become stable far from onset. There is unusual competition between twofold, threefold and fivefold symmetric structures in irreps $2 \pm, 3 \pm$ and $5 \pm$, between tetrahedral and threefold symmetry in irrep $4+$ and between tetrahedral, threefold and twofold symmetry in irrep 
4-. Irrep 1- gives rise, as expected, to a branch of solutions with icosahedral symmetry.

Interestingly, stable heteroclinic cycles between solutions with $\mathcal{D}_{2}^{z}$ symmetry appear to bifurcate supercritically from the origin for certain combinations of parameters in the case of irrep 5-. This can happen when all other known solutions bifurcate supercritically and are unstable, so these cycles might be expected to be observable in practice.

In nanocluster formation, a perfect Mackay icosahedron of 13 atoms is energetically preferred, but if an atom is gained the preferred structure has $\mathcal{D}_{3}^{z}$ or $\mathcal{D}_{2}^{z}$ symmetry (depending on the range of the interatomic force) [14] [15], and if one is lost the symmetry becomes $\mathcal{D}_{5}^{z}$ (or $\mathcal{D}_{4}^{d}$ for very short range interatomic forces). The same bifurcation to $\mathcal{D}_{3}^{z}$ on gaining an atom and $\mathcal{D}_{5}^{z}$ on losing an atom is shown at intermediate values of the interatomic force range for the 55 atom Mackay icosahedral cluster [14]. This behaviour is suggestive of a bifurcation with $\mathcal{I}_{h}$ governed by the $2-$ or 3 - irrep. When the 45 atom antiMackay icosahedron is preferred it loses symmetry to a $\mathcal{D}_{5}^{z}$ structure on losing an atom (again an irrep 2-/3- process), but on gaining one the resulting structure has only $\mathbb{Z}_{2}^{\text {refl }}$ symmetry, which is not predicted by theory in the general case.

Under certain circumstances [11] [12] an icosahedral solution is preferred at the primary bifurcation from a spherical base state, but is unstable. The final realised solution then may be one preferred at the bifurcation with icosahedral symmetry, with isotropy subgroup among those listed above. A further possibility, if the secondary bifurcation were governed by irrep $5-$, is that the end state might be a stable 
heteroclinic cycle.

Icosahedral viruses appear only to undergo configurational changes that respect the icosahedral symmetry, but as discussed above this may be an artefact of the imaging process. The results of this paper suggest that using an image reconstruction algorithm that respects the symmetry of the possible solution branches listed above may reveal symmetry-breaking behaviour related to virus function.

\section{Acknowledgements}

I would like to thank Ian Melbourne and Paul Matthews for helpful discussions. 


\section{Appendix}

The amplitude equations for irrep $4+$ to cubic order are

$$
\begin{aligned}
\dot{z}_{1}= & \lambda z_{1}-3 c_{1} z_{1}^{2}+2 c_{1} z_{1}\left(z_{2}+z_{3}+z_{4}\right) \\
& +c_{2} z_{1}^{3}+c_{3} z_{1}^{2}\left(z_{2}+z_{3}+z_{4}\right) \\
& +\left(3 c_{2}+4 c_{3}\right) z_{1}\left(z_{2}^{2}+z_{3}^{2}+z_{4}^{2}\right) \\
& -\left(3 c_{2}+5 c_{3}\right) z_{1}\left(z_{2} z_{3}+z_{3} z_{4}+z_{4} z_{2}\right), \\
\dot{z}_{2}= & \lambda z_{2}-3 c_{1} z_{2}^{2}+2 c_{1} z_{2}\left(z_{3}+z_{4}+z_{1}\right) \\
& +c_{2} z_{2}^{3}+c_{3} z_{2}^{2}\left(z_{3}+z_{4}+z_{1}\right) \\
& +\left(3 c_{2}+4 c_{3}\right) z_{2}\left(z_{3}^{2}+z_{4}^{2}+z_{1}^{2}\right) \\
& -\left(3 c_{2}+5 c_{3}\right) z_{2}\left(z_{3} z_{4}+z_{4} z_{1}+z_{1} z_{3}\right), \\
\dot{z}_{3}= & \lambda z_{3}-3 c_{1} z_{3}^{2}+2 c_{1} z_{3}\left(z_{4}+z_{1}+z_{2}\right) \\
& +c_{2} z_{3}^{3}+c_{3} z_{3}^{2}\left(z_{4}+z_{1}+z_{2}\right) \\
& +\left(3 c_{2}+4 c_{3}\right) z_{3}\left(z_{4}^{2}+z_{1}^{2}+z_{2}^{2}\right) \\
& -\left(3 c_{2}+5 c_{3}\right) z_{3}\left(z_{4} z_{1}+z_{1} z_{2}+z_{2} z_{4}\right), \\
\dot{z}_{4}= & \lambda z_{4}-3 c_{1} z_{4}^{2}+2 c_{1} z_{4}\left(z_{1}+z_{2}+z_{3}\right) \\
& +c_{2} z_{4}^{3}+c_{3} z_{4}^{2}\left(z_{1}+z_{2}+z_{3}\right) \\
& +\left(3 c_{2}+4 c_{3}\right) z_{4}\left(z_{1}^{2}+z_{2}^{2}+z_{3}^{2}\right) \\
& -\left(3 c_{2}+5 c_{3}\right) z_{4}\left(z_{1} z_{2}+z_{2} z_{3}+z_{3} z_{1}\right) .
\end{aligned}
$$

where the coefficients $c_{i}$ are distinct from those found in the one or three-dimensional cases. The amplitude equations for irrep 4- are the same as those for irrep $4+$ with the quadratic terms omitted. 
For irrep $5+$ the amplitude equations to cubic order are

$$
\begin{aligned}
& \dot{z}_{1}=\lambda z_{1}+c_{1} z_{1}\left(2 z_{1}-z_{2}-z_{3}-z_{4}-z_{5}\right) \\
& +c_{2}\left(z_{1}^{2}-z_{1}\left(z_{2}+z_{5}\right)+\left(z_{5}-z_{2}\right)\left(z_{3}-z_{4}\right)\right) \\
& +c_{3}\left(6 z_{1}^{3}-4 z_{1}^{2}\left(z_{3}+z_{4}\right)-3 z_{1}^{2}\left(z_{5}+z_{2}\right)+z_{1}\left(z_{5}^{2}+z_{2}^{2}\right)+4 z_{1}\left(z_{3} z_{4}+z_{3} z_{5}+z_{2} z_{4}\right)\right. \\
& \left.+z_{2} z_{3}\left(z_{2}+z_{3}\right)+z_{4} z_{5}\left(z_{4}+z_{5}\right)-z_{3} z_{5}\left(z_{3}+z_{5}\right)-z_{2} z_{4}\left(z_{2}+z_{4}\right)\right) \\
& +c_{4}\left(\frac{5}{2} z_{1}^{3}-\frac{3}{2} z_{1}^{2}\left(z_{2}+z_{3}+z_{4}+z_{5}\right)+z_{1}\left(z_{5}+z_{2}\right)\left(z_{3}+z_{4}\right)+z_{1}\left(z_{2} z_{5}+z_{3} z_{4}\right)\right) \\
& +c_{5} z_{1}\left(2 z_{1}^{2}-z_{1}\left(z_{2}+z_{3}+z_{4}+z_{5}\right)+z_{2}^{2}+z_{3}^{2}+z_{4}^{2}+z_{5}^{2}\right) \text {, } \\
& \dot{z}_{2}=\lambda z_{2}+c_{1} z_{2}\left(2 z_{2}-z_{3}-z_{4}-z_{5}-z_{1}\right) \\
& +c_{2}\left(z_{2}^{2}-z_{2}\left(z_{3}+z_{1}\right)+\left(z_{1}-z_{3}\right)\left(z_{4}-z_{5}\right)\right) \\
& +c_{3}\left(6 z_{2}^{3}-4 z_{2}^{2}\left(z_{4}+z_{5}\right)-3 z_{2}^{2}\left(z_{1}+z_{3}\right)+z_{2}\left(z_{1}^{2}+z_{3}^{2}\right)+4 z_{2}\left(z_{4} z_{5}+z_{4} z_{1}+z_{3} z_{5}\right)\right. \\
& \left.+z_{3} z_{4}\left(z_{3}+z_{4}\right)+z_{5} z_{1}\left(z_{5}+z_{1}\right)-z_{4} z_{1}\left(z_{4}+z_{1}\right)-z_{3} z_{5}\left(z_{3}+z_{5}\right)\right) \\
& +c_{4}\left(\frac{5}{2} z_{2}^{3}-\frac{3}{2} z_{2}^{2}\left(z_{3}+z_{4}+z_{5}+z_{1}\right)+z_{2}\left(z_{1}+z_{3}\right)\left(z_{4}+z_{5}\right)+z_{2}\left(z_{3} z_{1}+z_{4} z_{5}\right)\right) \\
& +c_{5} z_{2}\left(2 z_{2}^{2}-z_{2}\left(z_{3}+z_{4}+z_{5}+z_{1}\right)+z_{3}^{2}+z_{4}^{2}+z_{5}^{2}+z_{1}^{2}\right) \text {, } \\
& \dot{z}_{3}=\lambda z_{3}+c_{1} z_{3}\left(2 z_{3}-z_{4}-z_{5}-z_{1}-z_{2}\right) \\
& +c_{2}\left(z_{3}^{2}-z_{3}\left(z_{4}+z_{2}\right)+\left(z_{2}-z_{4}\right)\left(z_{5}-z_{1}\right)\right) \\
& +c_{3}\left(6 z_{3}^{3}-4 z_{3}^{2}\left(z_{5}+z_{1}\right)-3 z_{3}^{2}\left(z_{2}+z_{4}\right)+z_{3}\left(z_{2}^{2}+z_{4}^{2}\right)+4 z_{3}\left(z_{5} z_{1}+z_{5} z_{2}+z_{4} z_{1}\right)\right. \\
& \left.+z_{4} z_{5}\left(z_{4}+z_{5}\right)+z_{1} z_{2}\left(z_{1}+z_{2}\right)-z_{5} z_{2}\left(z_{5}+z_{2}\right)-z_{4} z_{1}\left(z_{4}+z_{1}\right)\right) \\
& +c_{4}\left(\frac{5}{2} z_{3}^{3}-\frac{3}{2} z_{3}^{2}\left(z_{4}+z_{5}+z_{1}+z_{2}\right)+z_{3}\left(z_{2}+z_{4}\right)\left(z_{5}+z_{1}\right)+z_{3}\left(z_{4} z_{2}+z_{5} z_{1}\right)\right) \\
& +c_{5} z_{3}\left(2 z_{3}^{2}-z_{3}\left(z_{4}+z_{5}+z_{1}+z_{2}\right)+z_{4}^{2}+z_{5}^{2}+z_{1}^{2}+z_{2}^{2}\right), \\
& \dot{z}_{4}=\lambda z_{4}+c_{1} z_{4}\left(2 z_{4}-z_{5}-z_{1}-z_{2}-z_{3}\right) \\
& +c_{2}\left(z_{4}^{2}-z_{4}\left(z_{5}+z_{3}\right)+\left(z_{3}-z_{5}\right)\left(z_{1}-z_{2}\right)\right) \\
& +c_{3}\left(6 z_{4}^{3}-4 z_{4}^{2}\left(z_{1}+z_{2}\right)-3 z_{4}^{2}\left(z_{3}+z_{5}\right)+z_{4}\left(z_{3}^{2}+z_{5}^{2}\right)+4 z_{4}\left(z_{1} z_{2}+z_{1} z_{3}+z_{5} z_{2}\right)\right. \\
& \left.+z_{5} z_{1}\left(z_{5}+z_{1}\right)+z_{2} z_{3}\left(z_{2}+z_{3}\right)-z_{1} z_{3}\left(z_{1}+z_{3}\right)-z_{5} z_{2}\left(z_{5}+z_{2}\right)\right) \\
& +c_{4}\left(\frac{5}{2} z_{4}^{3}-\frac{3}{2} z_{4}^{2}\left(z_{5}+z_{1}+z_{2}+z_{3}\right)+z_{4}\left(z_{3}+z_{5}\right)\left(z_{1}+z_{2}\right)+z_{4}\left(z_{5} z_{3}+z_{1} z_{2}\right)\right) \\
& +c_{5} z_{4}\left(2 z_{4}^{2}-z_{4}\left(z_{5}+z_{1}+z_{2}+z_{3}\right)+z_{5}^{2}+z_{1}^{2}+z_{2}^{2}+z_{3}^{2}\right),
\end{aligned}
$$




$$
\begin{aligned}
\dot{z}_{5}= & \lambda z_{5}+c_{1} z_{5}\left(2 z_{5}-z_{1}-z_{2}-z_{3}-z_{4}\right) \\
& +c_{2}\left(z_{5}^{2}-z_{5}\left(z_{1}+z_{4}\right)+\left(z_{4}-z_{1}\right)\left(z_{2}-z_{3}\right)\right) \\
& +c_{3}\left(6 z_{5}^{3}-4 z_{5}^{2}\left(z_{2}+z_{3}\right)-3 z_{5}^{2}\left(z_{4}+z_{1}\right)+z_{5}\left(z_{4}^{2}+z_{1}^{2}\right)+4 z_{5}\left(z_{2} z_{3}+z_{2} z_{4}+z_{1} z_{3}\right)\right. \\
& \left.+z_{1} z_{2}\left(z_{1}+z_{2}\right)+z_{3} z_{4}\left(z_{3}+z_{4}\right)-z_{2} z_{4}\left(z_{2}+z_{4}\right)-z_{1} z_{3}\left(z_{1}+z_{3}\right)\right) \\
& +c_{4}\left(\frac{5}{2} z_{5}^{3}-\frac{3}{2} z_{5}^{2}\left(z_{1}+z_{2}+z_{3}+z_{4}\right)+z_{5}\left(z_{4}+z_{1}\right)\left(z_{2}+z_{3}\right)+z_{5}\left(z_{1} z_{4}+z_{2} z_{3}\right)\right) \\
& +c_{5} z_{5}\left(2 z_{5}^{2}-z_{5}\left(z_{1}+z_{2}+z_{3}+z_{4}\right)+z_{1}^{2}+z_{2}^{2}+z_{3}^{2}+z_{4}^{2}\right),
\end{aligned}
$$

where the coefficients $c_{i}$ are distinct from those found in the one, three or four-dimensional cases. The amplitude equations for irrep 5- are the same as those for irrep $5+$ with the quadratic terms omitted.

\section{References}

[1] D.S. Goodsell and A.J. Olson (2000) "Structural symmetry and protein function" Annu. Rev. Biophys. Biomol. Struct. 29, 105153.

[2] F. Tama and C.L. Brooks III (2002) "The mechanism and pathway of $\mathrm{pH}$ induced swelling in cowpea chlorotic mottle virus" $J$. Mol. Biol. 318, 733-747.

[3] A.C. Steven, B.L. Trus, F.P. Booy, N. Cheng, A. Zlotnick, J.R. Caston and J.F. Conway (1997) "The making and breaking of symmetry in virus capsid assembly: glimpses of capsid biology from cryoelectron microscopy" FASEB J. 11, 733-742.

[4] B.L. Trus, F.P. Booy, W.W. Newcomb, J.C. Brown, F.L. Homa, D.R. Thomsen and A.C. Steven (1996) "The herpes simplex virus procapsid: structure, conformational changes upon maturation, 
and roles of the triplex proteins VP19c and VP23 in assembly" J. Mol. Biol. 263, 447-462.

[5] J.F. Conway, R.L. Duda, N. Cheng, R.W. Hendrix and A.C. Steven (1995) "Proteolytic and conformational control of virus capsid maturation: the bacteriophage HK97 system" J. Mol. Biol. 253, 86-99.

[6] J.F. Conway, W.R. Wikoff, N. Cheng, R.L. Duda, R.W. Hendrix, J.E. Johnson and A.C. Steven (2001) "Virus maturation involving large subunit rotations and local refolding" Science 292, 744-748.

[7] M.A. Canady, M. Tihova, T.N. Hanzlik, J.E. Johnson and M. Yeager (2000) 299, 573-584.

[8] D. Shechtman, I. Blech, D. Gratias and J.W. Cahn (1984) "Metallic phase with long-range orientational order and no translational symmetry" Phy. Rev. Lett. 53, 1951-1953.

[9] E. Ihrig and M. Golubitsky (1984) "Pattern Selection with $\mathrm{O}(3)$ symmetry", Physica D 13, 1-33.

[10] P. Chossat, R. Lauterbach and I. Melbourne (1990) "Steady state bifurcation with $\mathrm{O}(3)$ symmetry" Arch. Rational Mech. Anal. 113, 313-376.

[11] P.C. Matthews (2003) "Pattern formation on a sphere" Phys. Rev. E 67, 036206.

[12] P.C. Matthews (2003) "Transcritical bifurcation with O(3) symmetry" Nonlinearity 16, 1449-1471.

[13] H.M. Byrne and P.C. Matthews (2002) "Asymmetric growth of avascular solid tumours: exploiting symmetries" IMA J. Math. Appl. Med. Biol. 19, 1-29. 
[14] J.P.K. Doye and D.J. Wales (1997) "Structural consequences of the range of the interatomic potential: a menagerie of clusters" J. Chem. Soc., Faraday Trans. 93, 4233-4244; and references therein.

[15] J.P.K. Doye, D.J.Wales, W. Branz and F. Calvo (2001) "Modeling the structure of clusters of $\left(\mathrm{C}_{60}\right)$ molecules" Phys. Rev. B 64, 235409 .

[16] P. Kramer and R.W. Haase (1989) "Group Theory of Icosahedral Quasicrystals" in Introduction to the mathematics of Quasicrystals edited by M.V. Jaric (Academic Press).

[17] J.F. Cornwell, (1984) "Group Theory in Physics" (Academic, London).

[18] M. Golubitsky, I. Stewart and D.G. Schaeffer (1988) "Singularities and Groups in Bifurcation Theory, Volume II" (SpringerVerlag, New York).

[19] R. Lauterbach (1988) Habilitationsschrift "Problems with Spherical Symmetry: Studies on Bifurcations and Dynamics for O(3)Equivariant Equations."

[20] P.C. Matthews (2003) "Bifurcation in two-dimensional fixed point subspaces" arXiv:math.DS/0305392. 


\section{Figure Captions}

Figure 1: The generators of the icosahedral group

Figure 2: The lattice of inclusion of subgroups for the icosahedral group with reflections, $\mathcal{I}_{h}$.

Figure 3: Solutions with isotropy subgroups a) $\mathcal{I}_{h}$, b) $\mathcal{I}$ c) $\mathcal{T}$ and d) $\mathcal{T} \times \mathbb{Z}_{2}^{c}$

Figure 4: Solutions with isotropy subgroups a) $\mathcal{D}_{5} \times \mathbb{Z}_{2}^{c}$, b) $\mathcal{D}_{5}$, c) $\mathbb{Z}_{5} \times \mathbb{Z}_{2}^{c}$ and d) $\mathcal{D}_{5}^{z}$.

Figure 5: Solutions with isotropy subgroups a) $\mathcal{D}_{3} \times \mathbb{Z}_{2}^{c}$, b) $\mathcal{D}_{3}$, c) $\mathbb{Z}_{3} \times \mathbb{Z}_{2}^{c}$ and d) $\mathcal{D}_{3}^{z}$.

Figure 6: Solutions with isotropy subgroups a) $\mathcal{D}_{2} \times \mathbb{Z}_{2}^{c}$, b) $\mathcal{D}_{2}$, c) $\mathbb{Z}_{2} \times \mathbb{Z}_{2}^{c}$ and d) $\mathcal{D}_{2}^{z}$.

Figure 7: Partial bifurcation diagram for irrep 4+. Only solution branches 1 and 1' $\left(\mathcal{T} \times \mathbb{Z}_{2}^{c}\right.$ symmetry) and 2 and 2' $\left(\mathcal{D}_{3} \times \mathbb{Z}_{2}^{c}\right.$ symmetry), defined in table 6 , are shown. The amplitude $z$ of the solution is plotted against the bifurcation parameter $\lambda$. Stable solutions are shown by solid lines and unstable solutions by dotted lines. The diagram is shown for the case $c_{1}=0.5, c_{2}=-1, c_{3}=0.7$.

Figure 8: The lattice of isotropy subgroups for irrep $5+$. Inclusion is shown by an arrow.

Figure 9: Partial bifurcation diagram for irrep 5+. Only solution branches 1 and $1^{\prime}\left(\mathcal{D}_{3} \times \mathbb{Z}_{2}^{c}\right.$ symmetry $)$ and 2 and $2^{\prime}\left(\mathcal{D}_{5} \times \mathbb{Z}_{2}^{c}\right.$ symmetry), defined in table 8 , are shown. The amplitude $z$ of the solution is plotted against the bifurcation parameter $\lambda$. Stable solutions are shown by solid lines and unstable solutions by dotted lines. The diagram is shown for the case $c_{1}=c_{2}=-c_{3}=-c_{4}=-c_{5}=1$.

Figure 10: The lattice of isotropy subgroups for irrep 5-. Inclusion 
is shown by an arrow.

Figure 11: A heteroclinic cycle in the 5 - irrep in the case $\left(14 c_{3}+\right.$ $\left.5 c_{4}+2 c_{5}\right)\left(8 c_{3}+5 c_{4}+2 c_{5}\right)<0, \lambda>0$ and $5 c_{3}+2 c_{4}+2 c-5>0$. The arrows show the direction of time for the case $-\left(5 c_{4}+2 c_{5}\right) / 8<c_{3}<0$, and would be reversed in the case $0<c_{3}<-\left(5 c_{4}+2 c_{5}\right) / 8$. 


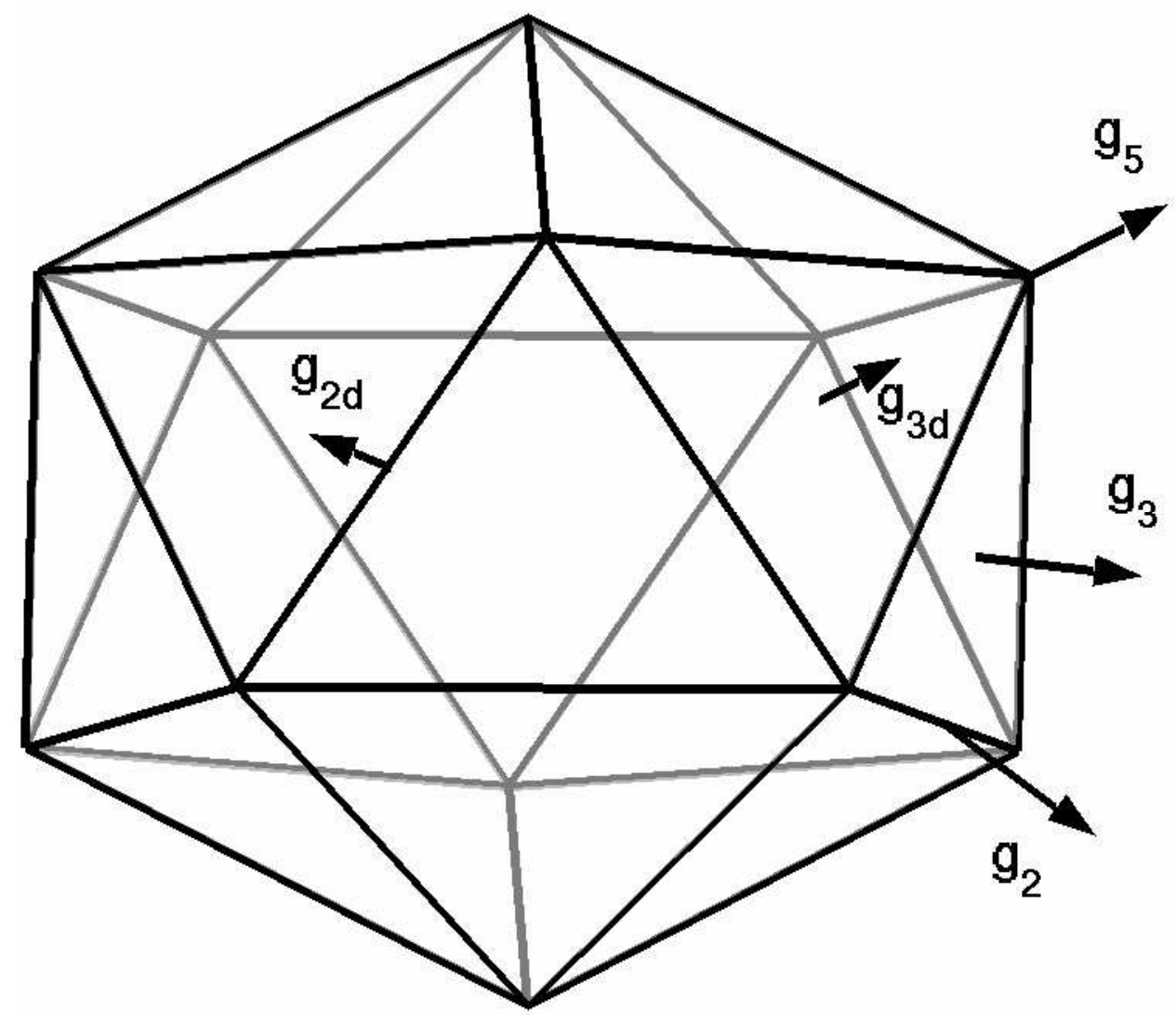

Figure 1: The generators of the icosahedral group 


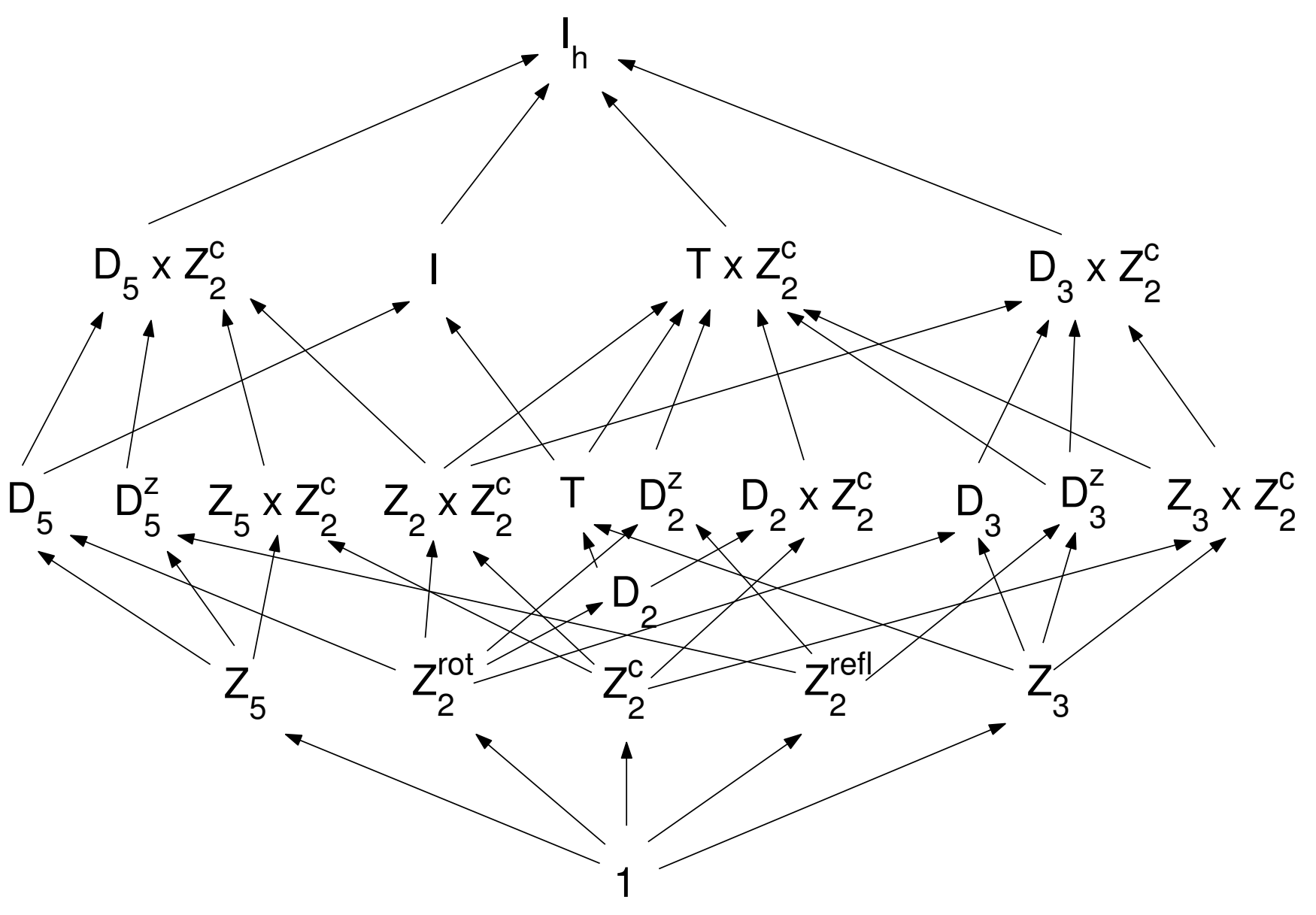

Figure 2: The lattice of inclusion of subgroups for the icosahedral group with reflections, $\mathcal{I}_{h}$. 


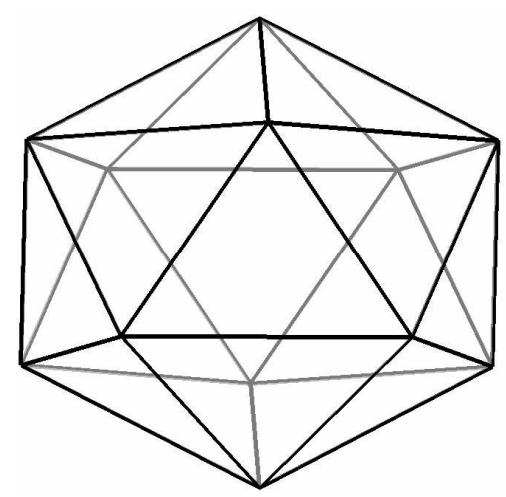

(a)

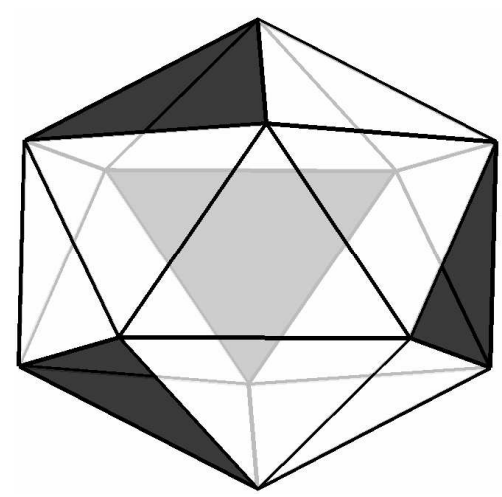

(c)

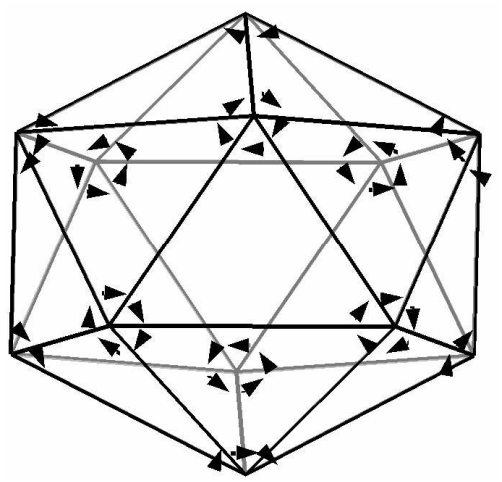

(b)

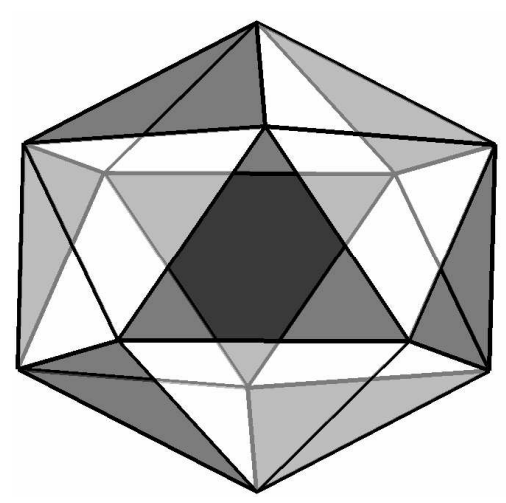

(d)

Figure 3: Solutions with isotropy subgroups a) $\mathcal{I}_{h}$, b) $\mathcal{I}$ c) $\mathcal{T}$ and d) $\mathcal{T} \times \mathbb{Z}_{2}^{c}$. 


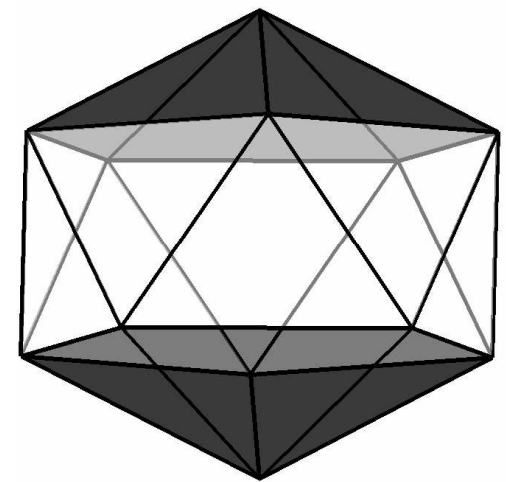

(a)

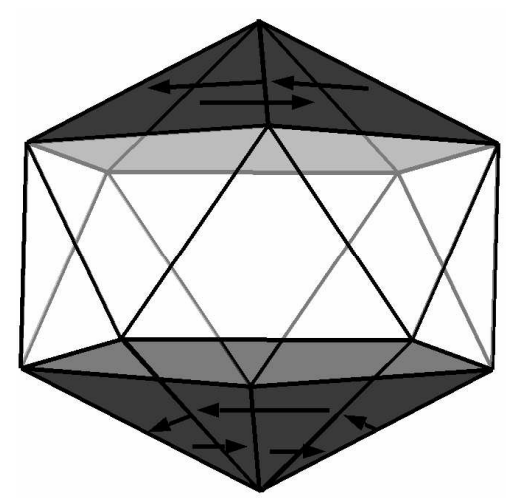

(c)

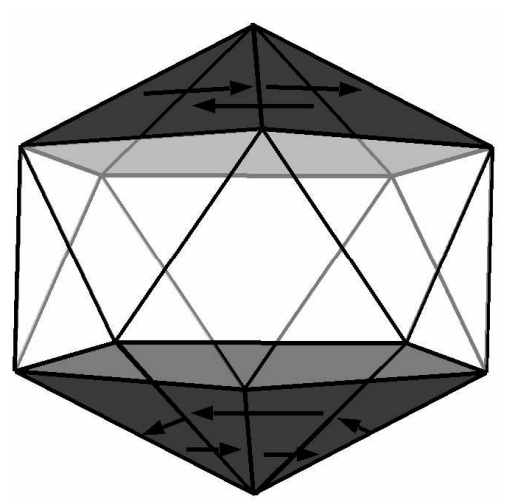

(b)

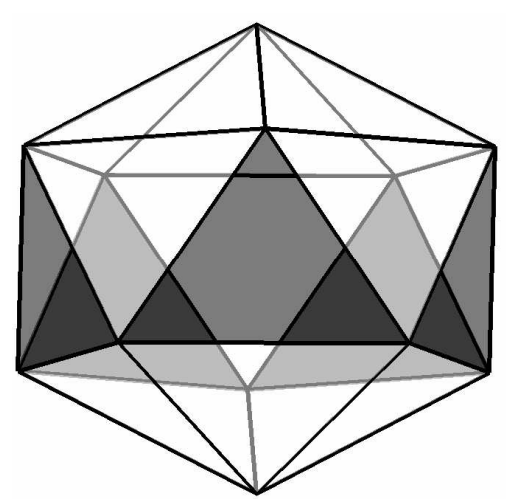

(d)

Figure 4: Solutions with isotropy subgroups a) $\mathcal{D}_{5} \times \mathbb{Z}_{2}^{c}$, b) $\mathcal{D}_{5}$, c) $\mathbb{Z}_{5} \times \mathbb{Z}_{2}^{c}$ and d) $\mathcal{D}_{5}^{z}$. 


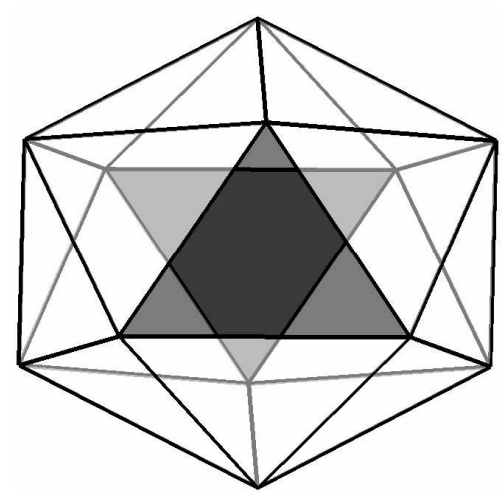

(a)

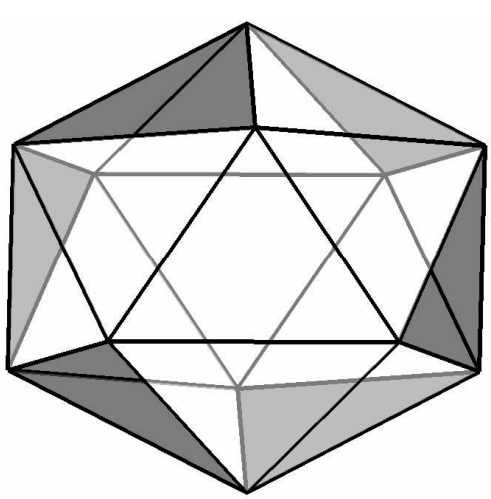

(c)

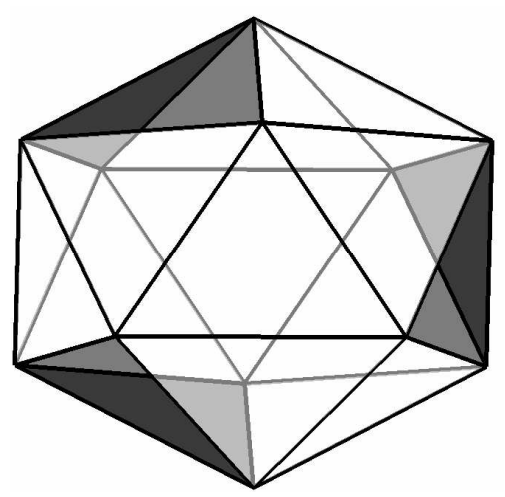

(b)

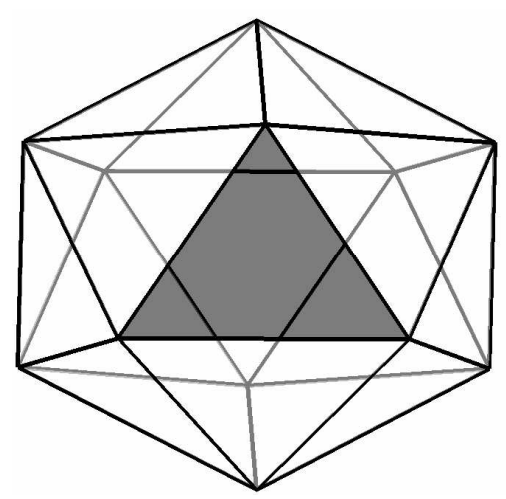

(d)

Figure 5: Solutions with isotropy subgroups a) $\mathcal{D}_{3} \times \mathbb{Z}_{2}^{c}$, b) $\mathcal{D}_{3}$, c) $\mathbb{Z}_{3} \times \mathbb{Z}_{2}^{c}$ and d) $\mathcal{D}_{3}^{z}$. 


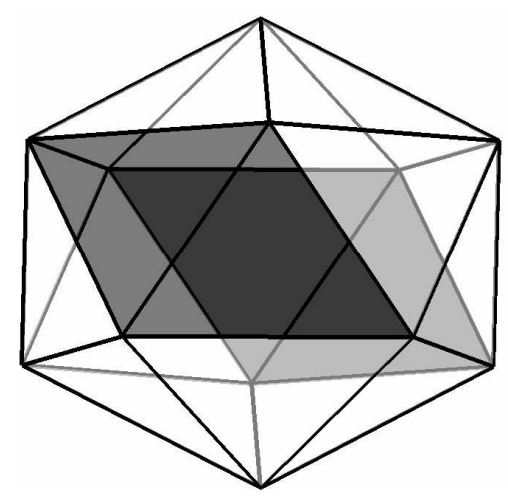

(a)

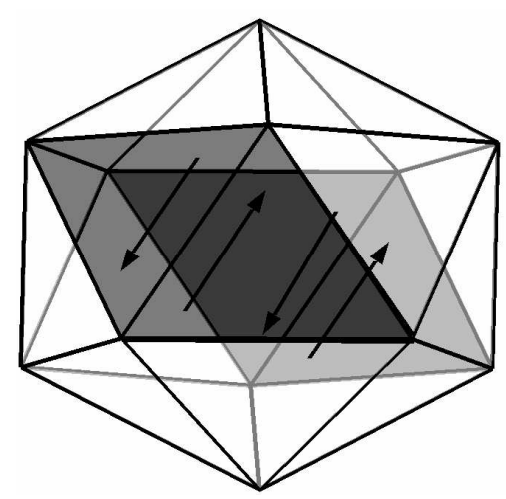

(c)

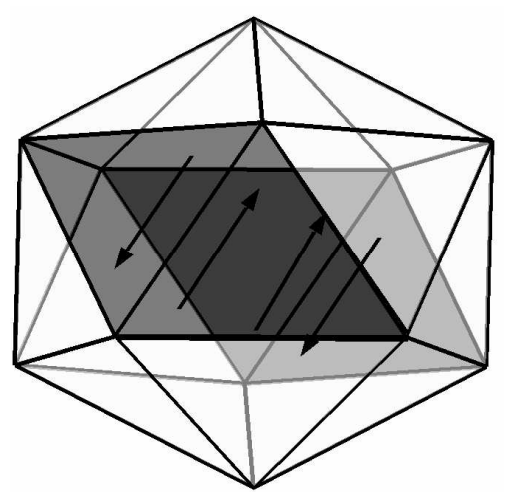

(b)

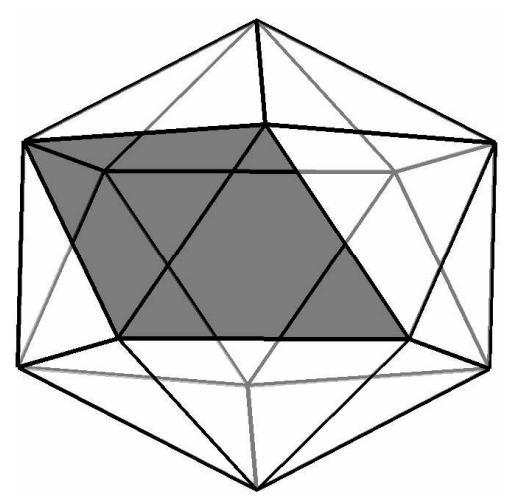

(d)

Figure 6: Solutions with isotropy subgroups a) $\mathcal{D}_{2} \times \mathbb{Z}_{2}^{c}$, b) $\mathcal{D}_{2}$, c) $\mathbb{Z}_{2} \times \mathbb{Z}_{2}^{c}$ and d) $\mathcal{D}_{2}^{z}$. 


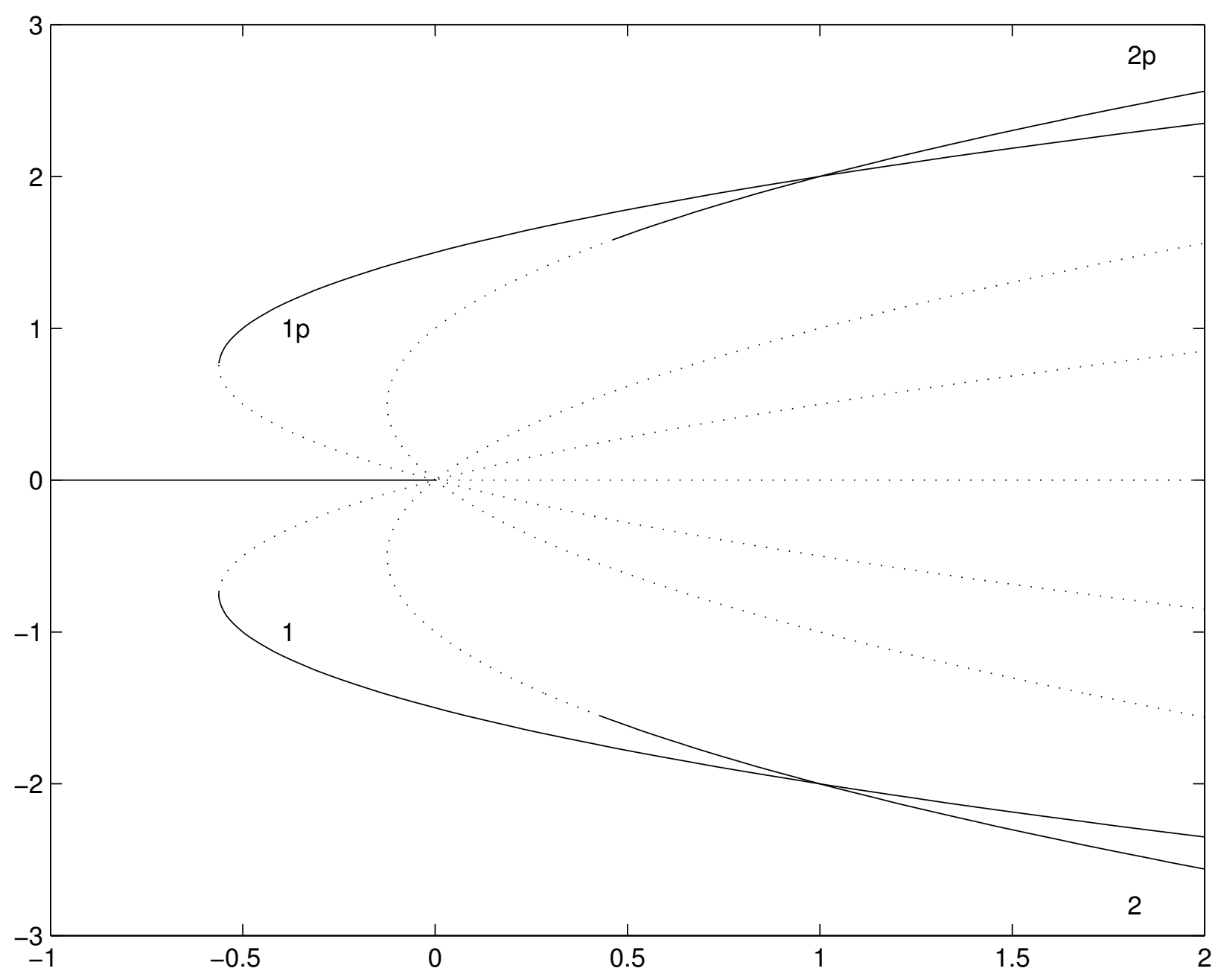

Figure 7: Partial bifurcation diagram for irrep 4+. Only solution branches 1 and $1^{\prime}\left(\mathcal{T} \times \mathbb{Z}_{2}^{c}\right.$ symmetry) and 2 and $2^{\prime}\left(\mathcal{D}_{3} \times \mathbb{Z}_{2}^{c}\right.$ symmetry $)$, defined in table 6 , are shown. The amplitude $z$ of the solution is plotted against the bifurcation parameter $\lambda$. Stable solutions are shown by solid lines and unstable solutions by dotted lines. The diagram is shown for the case $c_{1}=0.5, c_{2}=-1$, $c_{3}=0.7$. 


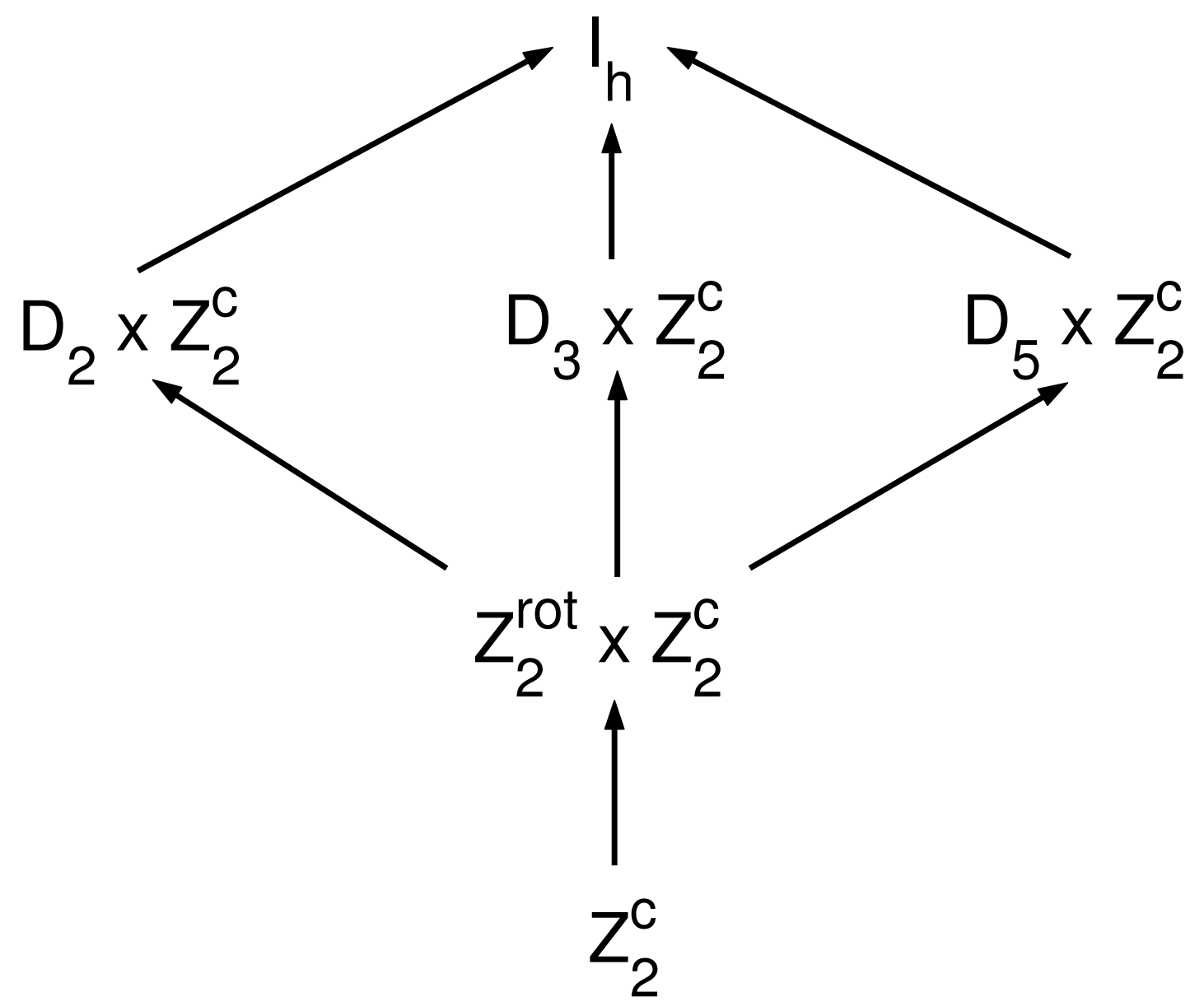

Figure 8: The lattice of isotropy subgroups for irrep $5+$. Inclusion is shown by an arrow. 


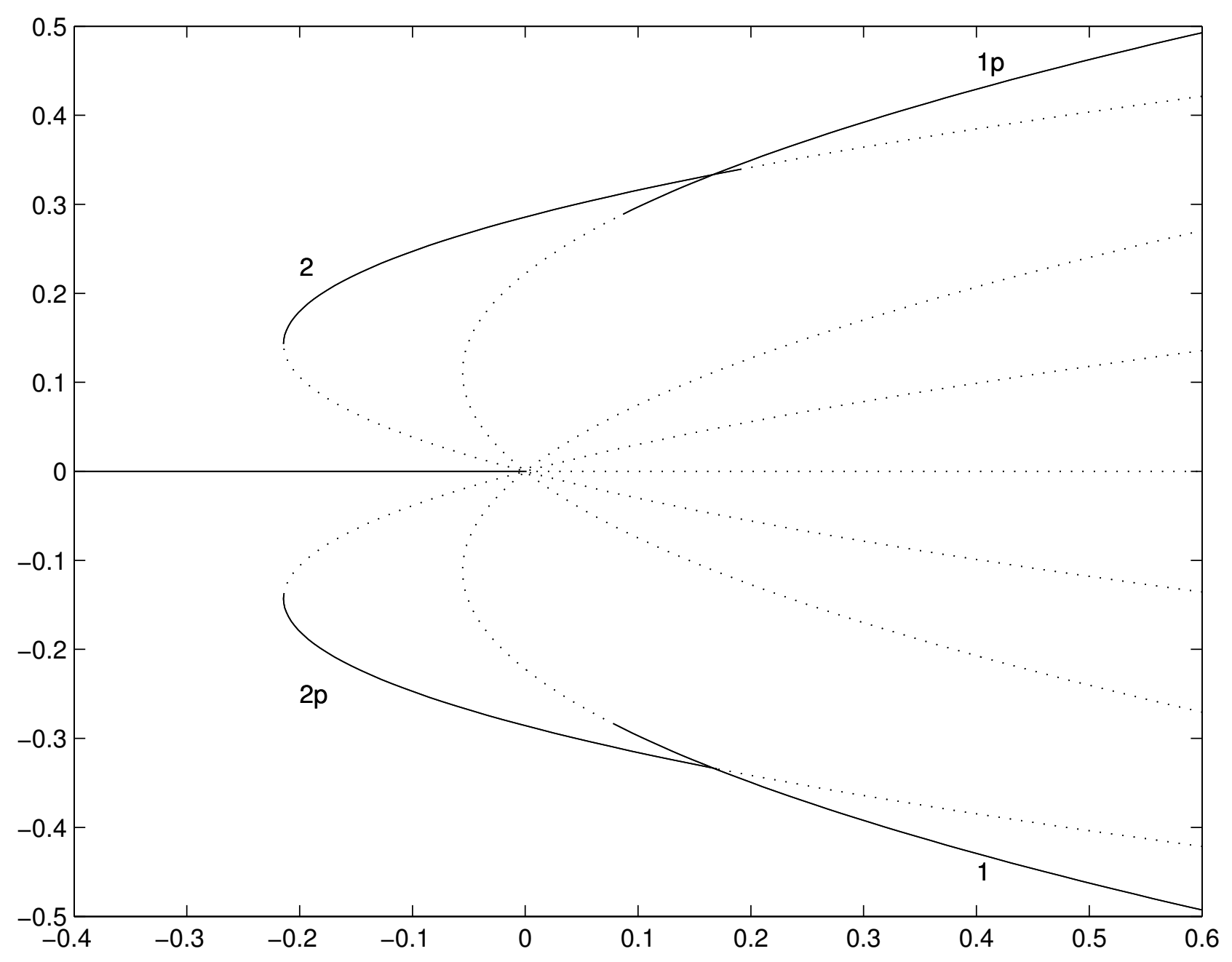

Figure 9: Partial bifurcation diagram for irrep 5+. Only solution branches 1 and $1^{\prime}\left(\mathcal{D}_{3} \times \mathbb{Z}_{2}^{c}\right.$ symmetry) and 2 and $2^{\prime}\left(\mathcal{D}_{5} \times \mathbb{Z}_{2}^{c}\right.$ symmetry), defined in table 8 , are shown. The amplitude $z$ of the solution is plotted against the bifurcation parameter $\lambda$. Stable solutions are shown by solid lines and unstable solutions by dotted lines. The diagram is shown for the case $c_{1}=$ $c_{2}=-c_{3}=-c_{4}=-c_{5}=1$. 


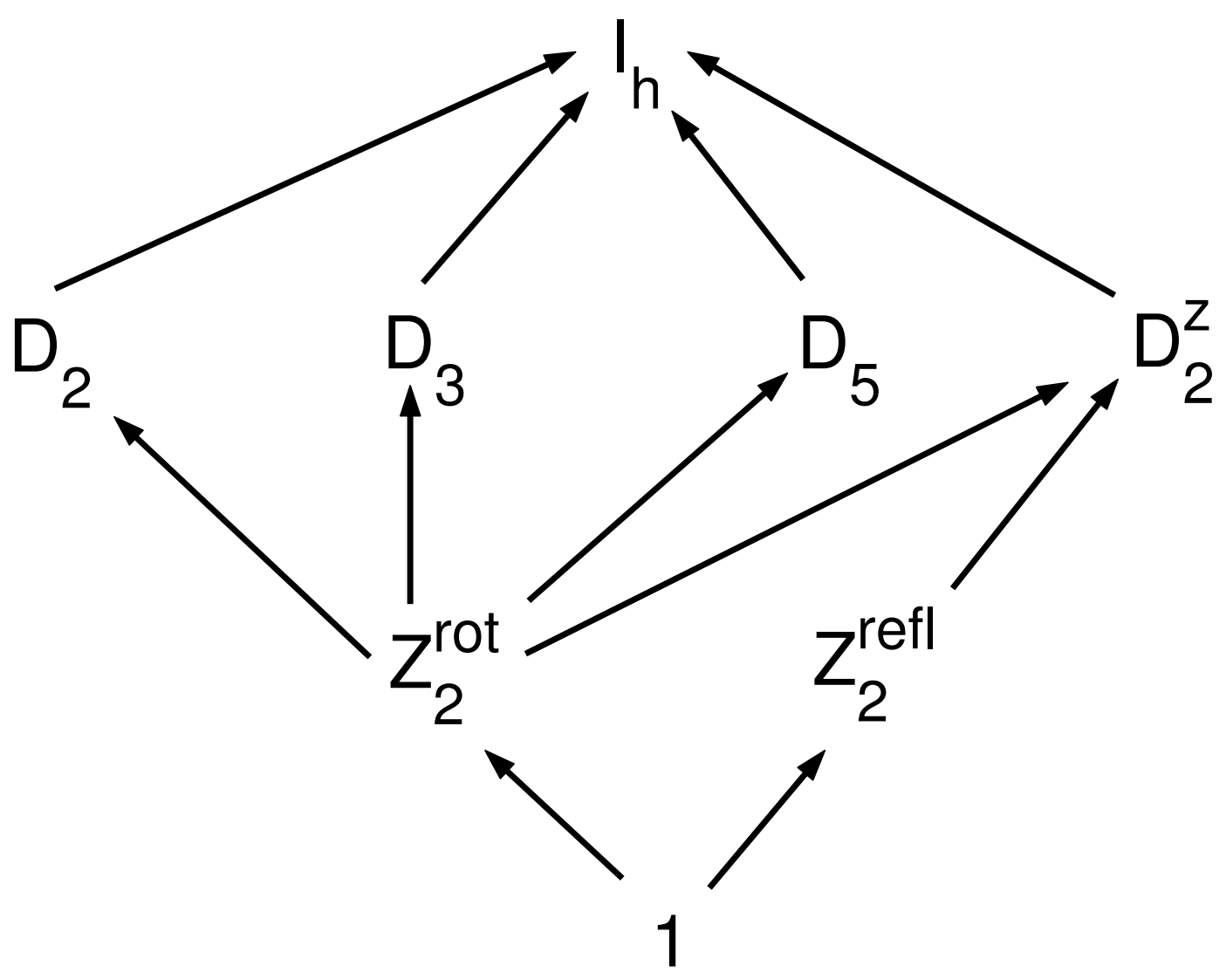

Figure 10: The lattice of isotropy subgroups for irrep 5-. Inclusion is shown by an arrow. 


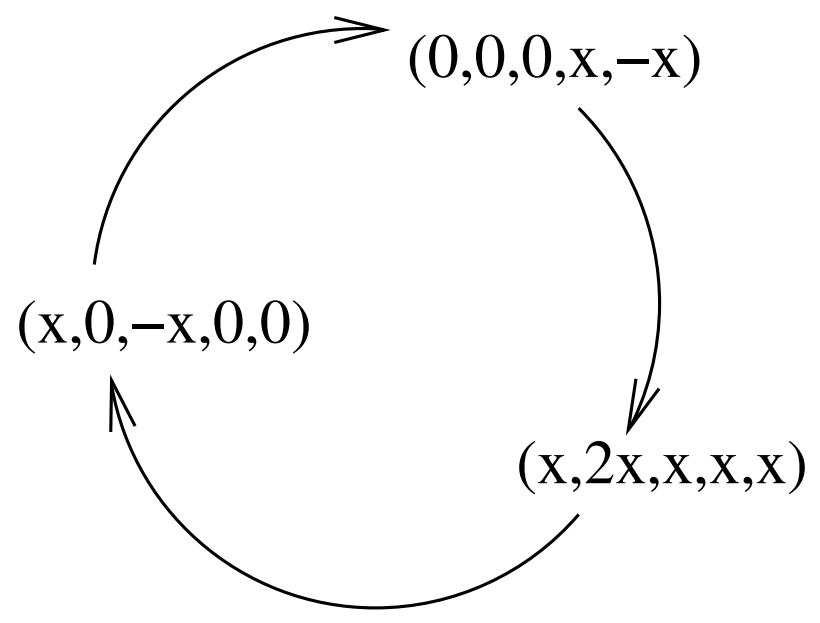

Figure 11: A heteroclinic cycle in the $5-$ irrep in the case $\left(14 c_{3}+5 c_{4}+\right.$ $\left.2 c_{5}\right)\left(8 c_{3}+5 c_{4}+2 c_{5}\right)<0, \lambda>0$ and $5 c_{3}+2 c_{4}+2 c-5>0$. The arrows show the direction of time for the case $-\left(5 c_{4}+2 c_{5}\right) / 8<c_{3}<0$, and would be reversed in the case $0<c_{3}<-\left(5 c_{4}+2 c_{5}\right) / 8$. 


\section{Table Captions}

Table 1: Character table for $\mathcal{I}_{h}$, where $\tau=(\sqrt{5}+1) / 2$.

Table 2: The subgroups of $\mathcal{I}_{h}$. Note that $\mathbb{Z}_{2}^{\text {rot }}, \mathbb{Z}_{2}^{\text {refl }}$ and $\mathbb{Z}_{2}^{c}$ are isomorphic, as are $\mathcal{D}_{n}$ and $\mathcal{D}_{n}^{z}$ for $n=2,3,5$.

Table 3: Axial isotropy subgroups for each nontrivial irrep of $\mathcal{I}_{h}$. Maximal, but non-axial, isotropy subgroups are given in brackets.

Table 4: Solution branches bifurcating at $\lambda=0$ for irreps $2 \pm$. Solutions for irreps $3 \pm$ can be found by changing the sign of $\sqrt{5}$ wherever it appears. Isotropy subgroup generators are given for the $2 \pm$ cases only. Solutions obtained by cyclic permutation of the $z_{i}$ are also permissible.

Table 5: Small- $\lambda$ approximations to the branches shown in Table 4 for irreps $2 \pm$. For irreps $3 \pm$ change the sign in front of $\sqrt{5}$ wherever it occurs.

Table 6: Solution branches bifurcating at $\lambda=0$ for irrep $4+$. Solutions obtained by interchanging any of the $z_{i}$ are also permissible.

Table 7: Solution branches bifurcating at $\lambda=0$ for irrep 4-. Solutions obtained by interchanging any of the $z_{i}$ are also permissible.

Table 8: Representative solution branches bifurcating at $\lambda=0$ for irrep $5+$.

Table 9: Representative solution branches bifurcating at $\lambda=0$ for irrep 5-.

Table 10: Axial isotropy subgroups for each nontrivial irrep of $\mathcal{I}$. Maximal, but non-axial, isotropy subgroups are given in brackets. 


\begin{tabular}{|c|c|c|c|c|c|c|c|c|c|c|}
\hline Irrep & $\begin{array}{l}C(e) \\
(1)\end{array}$ & $\begin{array}{l}C\left(g_{5}\right) \\
(12)\end{array}$ & $\begin{array}{l}C\left(g_{5}^{2}\right) \\
(12)\end{array}$ & $\begin{array}{l}C\left(g_{2}\right) \\
(15)\end{array}$ & $\begin{array}{l}C\left(g_{3}\right) \\
(20)\end{array}$ & $\begin{array}{l}C\left(g_{c}\right) \\
(1)\end{array}$ & $\begin{array}{l}C\left(g_{c} g_{5}\right) \\
(12)\end{array}$ & $\begin{array}{l}C\left(g_{c} g_{5}^{2}\right) \\
(12)\end{array}$ & $\begin{array}{l}C\left(g_{c} g_{2}\right) \\
(15)\end{array}$ & $\begin{array}{l}C\left(g_{c} g_{3}\right) \\
(20)\end{array}$ \\
\hline $1+$ & 1 & 1 & 1 & 1 & 1 & 1 & 1 & 1 & 1 & 1 \\
\hline 1- & 1 & 1 & 1 & 1 & 1 & -1 & -1 & -1 & -1 & -1 \\
\hline $2+$ & 3 & $\tau$ & $1-\tau$ & -1 & 0 & 3 & $\tau$ & $1-\tau$ & -1 & 0 \\
\hline $2-$ & 3 & $\tau$ & $1-\tau$ & -1 & 0 & -3 & $-\tau$ & $-1+\tau$ & 1 & 0 \\
\hline $3+$ & 3 & $1-\tau$ & $\tau$ & -1 & 0 & 3 & $1-\tau$ & $\tau$ & -1 & 0 \\
\hline $3-$ & 3 & $1-\tau$ & $\tau$ & -1 & 0 & -3 & $-1+\tau$ & $-\tau$ & 1 & 0 \\
\hline $4+$ & 4 & -1 & -1 & 0 & 1 & 4 & -1 & -1 & 0 & 1 \\
\hline 4- & 4 & -1 & -1 & 0 & 1 & -4 & 1 & 1 & 0 & -1 \\
\hline $5+$ & 5 & 0 & 0 & 1 & -1 & 5 & 0 & 0 & 1 & -1 \\
\hline 5- & 5 & 0 & 0 & 1 & -1 & -5 & 0 & 0 & -1 & 1 \\
\hline
\end{tabular}

Table 1: Character table for $\mathcal{I}_{h}$, where $\tau=(\sqrt{5}+1) / 2$. 


\begin{tabular}{|c|c|c|c|c|}
\hline Subgroup & Generators & Abstract definition & Elements & Order \\
\hline $\mathcal{I}$ & $g_{2}, g_{3}$ & $g_{2}^{2}=g_{3}^{3}=\left(g_{2} g_{3}\right)^{5}=e$ & $\begin{array}{l}g_{5}^{\mu} g_{2 d}^{\sigma}, g_{5}^{\mu} g_{2} g_{5}^{\nu} g_{2 d}^{\sigma} \\
\mu, \nu=0,1,2,3,4, \sigma=0,1\end{array}$ & 60 \\
\hline $\mathcal{T} \times \mathbb{Z}_{2}^{c}$ & $g_{2}, g_{3 d}, g_{c}$ & $g_{2}^{2}=g_{3 d}^{3}=\left(g_{2} g_{3 d}\right)^{3}=g_{c}^{2}=e$ & $\begin{array}{l}g_{3 d}^{\tau} g_{c}^{\sigma}, g_{3 d}^{\tau} g_{2} g_{3 d}^{\mu} g_{c}^{\sigma} \\
\left(\text { where } g_{3 d}=g_{5} g_{3} g_{5}^{-1}\right)\end{array}$ & 24 \\
\hline $\mathcal{D}_{5} \times \mathbb{Z}_{2}^{c}$ & $g_{2 d}, g_{5}, g_{c}$ & $g_{2 d}^{2}=g_{5}^{5}=\left(g_{5} g_{2 d}\right)^{2}=g_{c}^{2}=e$ & $\begin{array}{l}\tau, \mu=0,1,2, \sigma=0,1 \\
g_{5}^{\nu} g_{2 d}^{\sigma} g_{c}^{\tau} \\
\nu=0,1,2,3,4, \sigma, \tau=0,1\end{array}$ & 20 \\
\hline $\mathcal{D}_{3} \times \mathbb{Z}_{2}^{c}$ & $g_{2 d}, g_{3}, g_{c}$ & $g_{2 d}^{2}=g_{3}^{3}=\left(g_{3} g_{2 d}\right)^{2}=g_{c}^{2}=e$ & $\begin{array}{l}g_{3}^{\nu} g_{2 d}^{\sigma} g_{c}^{\tau} \\
\nu=0,1,2, \sigma, \tau=0,1\end{array}$ & 12 \\
\hline $\mathcal{T}$ & $g_{2}, g_{3 d}$ & $g_{2}^{2}=g_{3 d}^{3}=\left(g_{2} g_{3 d}\right)^{3}=e$ & $\begin{array}{l}g_{3 d}^{\tau}, g_{3 d}^{\tau} g_{2} g_{3 d}^{\mu} \\
\tau, \mu=0,1,2\end{array}$ & 12 \\
\hline $\mathcal{D}_{5}$ & $g_{2 d}, g_{5}$ & $g_{2 d}^{2}=g_{5}^{5}=\left(g_{5} g_{2 d}\right)^{2}=e$ & $\begin{array}{l}g_{5}^{\nu} g_{2 d}^{\sigma} \\
\nu=0,1,2,3,4, \sigma=0,1\end{array}$ & 10 \\
\hline $\mathcal{D}_{5}^{z}$ & $g_{c} g_{2 d}, g_{5}$ & $\left(g_{c} g_{2 d}\right)^{2}=g_{5}^{5}=\left(g_{5} g_{c} g_{2 d}\right)^{2}=e$ & $\begin{array}{l}g_{5}^{\nu}\left(g_{c} g_{2 d}\right)^{\sigma} \\
\nu=0,1,2,3,4, \sigma=0,1\end{array}$ & 10 \\
\hline $\mathbb{Z}_{5} \times \mathbb{Z}_{2}^{c}$ & $g_{c}, g_{5}$ & $g_{c}^{2}=g_{5}^{5}=e$ & $\begin{array}{l}g_{5}^{\nu} g_{c}^{\sigma} \\
\nu=0,1,2,3,4, \sigma=0,1\end{array}$ & 10 \\
\hline $\mathcal{D}_{2} \times \mathbb{Z}_{2}^{c}$ & $g_{2 d}, g_{2}, g_{c}$ & $g_{2 d}^{2}=g_{2}^{2}=\left(g_{2} g_{2 d}\right)^{2}=g_{c}^{2}=e$ & $\begin{array}{l}g_{2}^{\nu} g_{2 d}^{\sigma} g_{c}^{\tau} \\
\nu, \sigma, \tau=0,1\end{array}$ & 8 \\
\hline $\mathcal{D}_{3}$ & $g_{2 d}, g_{3}$ & $g_{2 d}^{2}=g_{3}^{3}=\left(g_{3} g_{2 d}\right)^{2}=e$ & $\begin{array}{l}g_{3}^{\nu} g_{2 d}^{\sigma} \\
\nu=0,1,2, \sigma=0,1\end{array}$ & 6 \\
\hline $\mathcal{D}_{3}^{z}$ & $g_{c} g_{2 d}, g_{3}$ & $\left(g_{c} g_{2 d}\right)^{2}=g_{3}^{3}=\left(g_{3} g_{c} g_{2 d}\right)^{2}=e$ & $\begin{array}{l}g_{3}^{\nu}\left(g_{c} g_{2 d}\right)^{\sigma} \\
\nu=0,1,2, \sigma=0,1\end{array}$ & 6 \\
\hline $\mathbb{Z}_{3} \times \mathbb{Z}_{2}^{c}$ & $g_{c}, g_{3}$ & $g_{c}^{2}=g_{3}^{3}=e$ & $\begin{array}{l}g_{3}^{\nu} g_{c}^{\sigma} \\
\nu=0,1,2, \sigma=0,1\end{array}$ & 6 \\
\hline $\mathbb{Z}_{5}$ & $g_{5}$ & $g_{5}^{5}=e$ & $\begin{array}{l}g_{5}^{\nu} \\
\nu=0,1,2,3,4\end{array}$ & 5 \\
\hline $\mathcal{D}_{2}$ & $g_{2 d}, g_{2}$ & $g_{2 d}^{2}=g_{2}^{2}=\left(g_{2} g_{2 d}\right)^{2}=e$ & $\begin{array}{l}g_{2}^{\nu} g_{2 d}^{\sigma} \\
\nu=0,1, \sigma=0,1\end{array}$ & 4 \\
\hline $\mathcal{D}_{2}^{z}$ & $g_{c} g_{2 d}, g_{2}$ & $\left(g_{c} g_{2 d}\right)^{2}=g_{2}^{2}=\left(g_{2} g_{c} g_{2 d}\right)^{2}=e$ & $\begin{array}{l}g_{2}^{\nu}\left(g_{c} g_{2 d}\right)^{\sigma} \\
\nu=0,1, \sigma=0,1\end{array}$ & 4 \\
\hline $\mathbb{Z}_{2} \times \mathbb{Z}_{2}^{c}$ & $g_{c}, g_{2}$ & $g_{c}^{2}=g_{2}^{2}=e$ & $\begin{array}{l}g_{2}^{\nu} g_{c}^{\sigma} \\
\nu=0,1, \sigma=0,1\end{array}$ & 4 \\
\hline $\mathbb{Z}_{3}$ & $g_{3}$ & $g_{3}^{3}=e$ & $\begin{array}{l}g_{3}^{\nu} \\
\nu=0,1,2\end{array}$ & 3 \\
\hline $\mathbb{Z}_{2}^{\text {rot }}$ & $g_{2}$ & $g_{2}^{2}=e$ & $\begin{array}{l}g_{2}^{\nu} \\
\nu=0,1\end{array}$ & 2 \\
\hline $\mathbb{Z}_{2}^{\text {refl }}$ & $g_{c} g_{2}$ & $\left(g_{c} g_{2}\right)^{2}=e$ & $\begin{array}{l}\left(g_{c} g_{2}\right)^{\nu} \\
\nu=0,1\end{array}$ & 2 \\
\hline $\mathbb{Z}_{2}^{c}$ & $g_{c}$ & $g_{c}^{2}=e$ & $\begin{array}{l}g_{c}^{\nu} \\
\nu=0,1\end{array}$ & 2 \\
\hline $\mathbb{1}$ & $e$ & - & $e$ & 1 \\
\hline
\end{tabular}

Table 2: The subgroups of $\mathcal{I}_{h}$. Note that $\mathbb{Z}_{2}^{\text {rot }}, \mathbb{Z}_{2}^{\text {refl }}$ and $\mathbb{Z}_{2}^{c}$ are isomorphic, as are $\mathcal{D}_{n}$ and $\mathcal{D}_{n}^{z}$ for $n=2,3,5$. 


\begin{tabular}{ll} 
Irrep & Axial (maximal) isotropy subgro \\
\hline 1- & $\mathcal{I}$ \\
2+ and 3+ & $\mathbb{Z}_{2} \times \mathbb{Z}_{2}^{c}, \mathbb{Z}_{3} \times \mathbb{Z}_{2}^{c}, \mathbb{Z}_{5} \times \mathbb{Z}_{2}^{c}$ \\
2- and 3- & $\mathcal{D}_{2}^{z}, \mathcal{D}_{3}^{z}, \mathcal{D}_{5}^{z}$ \\
$4+$ & $\mathcal{T} \times \mathbb{Z}_{2}^{c}, \mathcal{D}_{3} \times \mathbb{Z}_{2}^{c}$ \\
$4-$ & $\mathcal{T}, \mathcal{D}_{3}, \mathcal{D}_{3}^{z}, \mathcal{D}_{2}^{z}$ \\
$5+$ & $\mathcal{D}_{3} \times \mathbb{Z}_{2}^{c}, \mathcal{D}_{5} \times \mathbb{Z}_{2}^{c},\left(\mathcal{D}_{2} \times \mathbb{Z}_{2}^{c}\right)$ \\
$5-$ & $\mathcal{D}_{3}, \mathcal{D}_{5}, \mathcal{D}_{2}^{z},\left(\mathcal{D}_{2}\right)$
\end{tabular}

Table 3: Axial isotropy subgroups for each nontrivial irrep of $\mathcal{I}_{h}$. Maximal, but non-axial, isotropy subgroups are given in brackets.

\begin{tabular}{|c|c|c|c|}
\hline & Representative equations & Growth rate eigenvalues & $\begin{array}{l}\text { Isotropy subgroup } \\
\text { [generators] }\end{array}$ \\
\hline 1 & $\begin{array}{l}z_{1}=z_{2}=0 \\
\frac{1}{10}\left(5\left(c_{2}+c_{3}\right)-\sqrt{5}\left(c_{2}-c_{3}\right)\right) z_{3}^{4} \\
-c_{1} z_{3}^{2}+\lambda=0\end{array}$ & $\begin{array}{l}\lambda-c_{1} z_{3}^{2}+c_{2} z_{3}^{4}, \lambda-c_{1} z_{3}^{2}+c_{3} z_{3}^{4} \\
\lambda-3 c_{1} z_{3}^{2} \\
+\frac{1}{2}\left(5\left(c_{2}+c_{3}\right)-\sqrt{5}\left(c_{2}-c_{3}\right)\right) z_{3}^{4}\end{array}$ & $\begin{array}{l}\mathbb{Z}_{2} \times \mathbb{Z}_{2}^{c}\left[g_{2} g_{2 d}, g_{c}\right](2+) \\
\mathcal{D}_{2}^{z}\left[g_{2} g_{2 d}, g_{c} g_{2 d}\right](2-)\end{array}$ \\
\hline 2 & $\begin{array}{l}z_{1}=0, z_{2}^{2}=\frac{1}{2}(3-\sqrt{5}) z_{3}^{2} \\
\left(62 c_{2}+63 c_{3}-5 \sqrt{5} c_{3}\right) z_{3}^{4}- \\
10(\sqrt{5}+6) c_{1} z_{3}^{2}+(35+11 \sqrt{5}) \lambda=0\end{array}$ & $\begin{array}{l}\lambda+\frac{1}{2}(\sqrt{5}-5) c_{1} z_{3}^{2} \\
+\frac{1}{2}\left(15 c_{2}-7 \sqrt{5} c_{2}+2 \sqrt{5} c_{3}\right) z_{3}^{4}(\mathrm{x} 2) \\
\lambda+\frac{3}{2}(\sqrt{5}-5) c_{1} z_{3}^{2} \\
+\frac{1}{2}\left(35 c_{2}-11 \sqrt{5} c_{2}+40 c_{3}-14 \sqrt{5} c_{3}\right) z_{3}^{4}\end{array}$ & $\begin{array}{l}\mathbb{Z}_{5} \times \mathbb{Z}_{2}^{c}\left[g_{5}, g_{c}\right](2+) \\
\mathcal{D}_{5}^{z}\left[g_{5}, g_{c} g_{2 d}\right](2-)\end{array}$ \\
\hline 3 & $\begin{array}{l}z_{1}=0, z_{2}^{2}=\frac{1}{2}(7+3 \sqrt{5}) z_{3}^{2} \\
\left(142 c_{2}+263 c_{3}+99 \sqrt{5} c_{3}\right) z_{3}^{4}+ \\
(18 \sqrt{5}-120) c_{1} z_{3}^{2}+(75-29 \sqrt{5}) \lambda=0\end{array}$ & $\begin{array}{l}\lambda-\frac{3}{2}(\sqrt{5}+3) c_{1} z_{3}^{2} \\
+\frac{1}{2}\left(39 c_{2}+17 \sqrt{5} c_{2}+10 \sqrt{5} c_{3}+24 c_{3}\right) z_{3}^{4}(\mathrm{x} 2) \\
\lambda-\frac{9}{2}(\sqrt{5}+3) c_{1} z_{3}^{2} \\
+\frac{1}{2}\left(75 c_{2}+29 \sqrt{5} c_{2}+240 c_{3}+106 \sqrt{5} c_{3}\right) z_{3}^{4}\end{array}$ & $\begin{array}{l}\mathbb{Z}_{3} \times \mathbb{Z}_{2}^{c}\left[g_{3}, g_{c}\right](2+) \\
\mathcal{D}_{3}^{z}\left[g_{3}, g_{c} g_{2 d}\right](2-)\end{array}$ \\
\hline 4 & $\begin{array}{l}z_{1}^{2}=z_{2}^{2}=z_{3}^{2} \\
\left(142 c_{2}+263 c_{3}+99 \sqrt{5} c_{3}\right) z_{3}^{4}- \\
(33 \sqrt{5}+135) c_{1} z_{3}^{2}+(45+11 \sqrt{5}) \lambda=0\end{array}$ & $\begin{array}{l}\lambda-3 c_{1} z_{3}^{2} \\
+\frac{1}{2}\left(9\left(c_{2}+c_{3}\right)+\sqrt{5}\left(c_{2}-c_{3}\right)\right) z_{3}^{4}(\mathrm{x} 2) \\
\lambda-9 c_{1} z_{3}^{2} \\
+\frac{1}{2}\left(45\left(c_{2}+c_{3}\right)-11 \sqrt{5}\left(c_{2}-c_{3}\right)\right) z_{3}^{4}\end{array}$ & $\begin{array}{l}\mathbb{Z}_{3} \times \mathbb{Z}_{2}^{c}\left[g_{5} g_{2} g_{5}^{2} g_{2 d}, g_{c}\right](2+) \\
\mathcal{D}_{3}^{z}\left[g_{5} g_{2} g_{5}^{2} g_{2 d}, g_{c} g_{5}^{3} g_{2 d}\right](2-)\end{array}$ \\
\hline 5 & $\begin{array}{l}z_{1}^{2}=\frac{1}{2}(3-\sqrt{5}) z_{3}^{2}, z_{2}^{2}=\frac{1}{2}(3+\sqrt{5}) z_{3}^{2}, \\
8\left(5\left(c_{2}+c_{3}\right)-\sqrt{5}\left(c_{2}-c_{3}\right)\right) z_{3}^{4} \\
-20 c_{1} z_{3}^{2}+5 \lambda=0\end{array}$ & $\begin{array}{l}\lambda-4 c_{1} z_{3}^{2}+16 c_{2} z_{3}^{4}, \lambda-4 c_{1} z_{3}^{2}+16 c_{3} z_{3}^{4} \\
\lambda-12 c_{1} z_{3}^{2}+8\left(5\left(c_{2}+c_{3}\right)-\sqrt{5}\left(c_{2}-c_{3}\right)\right) z_{3}^{4}\end{array}$ & $\begin{array}{l}\mathbb{Z}_{2} \times \mathbb{Z}_{2}^{c}\left[g_{5}^{2} g_{2 d}, g_{c}\right](2+) \\
\mathcal{D}_{2}^{z}\left[g_{5}^{2} g_{2 d}, g_{c} g_{5} g_{2} g_{5} g_{2 d}\right](2-)\end{array}$ \\
\hline
\end{tabular}

Table 4: Solution branches bifurcating at $\lambda=0$ for irreps $2 \pm$. Solutions for irreps $3 \pm$ can be found by changing the sign of $\sqrt{5}$ wherever it appears. Isotropy subgroup generators are given for the $2 \pm$ cases only. Solutions obtained by cyclic permutation of the $z_{i}$ are also permissible. 


$$
\begin{array}{rlrl}
1 & z_{1}=z_{2}=0, & & \approx-\frac{\lambda^{2}}{10 c_{1}^{2}}\left(c_{2}-c_{3}\right)(5-\sqrt{5}), \frac{\lambda^{2}}{10 c_{1}^{2}}\left(c_{2}-c_{3}\right)(5 \\
z_{3}^{2} \approx \frac{\lambda}{c_{1}}+\frac{\lambda^{2}}{10 c_{1}^{3}}\left(5\left(c_{2}+c_{3}\right)-\sqrt{5}\left(c_{2}-c_{3}\right)\right) & & -2 \lambda+\frac{\lambda^{2}}{5 c_{1}^{2}}\left(5\left(c_{2}+c_{3}\right)-\sqrt{5}\left(c_{2}-c_{3}\right)\right) \\
2 & z_{1}=0, z_{2}^{2}=\frac{1}{2}(3-\sqrt{5}) z_{3}^{2}, & & \approx-\frac{8 \sqrt{5} \lambda^{2}}{25 c_{1}^{2}}\left(c_{2}-c_{3}\right)(\mathrm{x} 2), \\
z_{3}^{2} \approx \frac{\lambda}{10 c_{1}}(5+\sqrt{5}) & & -2 \lambda+\frac{\lambda^{2}}{25 c_{1}^{2}}\left(25\left(c_{2}+c_{3}\right)+\sqrt{5}\left(c_{2}-c_{3}\right)\right) \\
& +\frac{\lambda^{2}}{100 c_{1}^{3}}\left(5(5+\sqrt{5})\left(c_{2}+c_{3}\right)+(1+\sqrt{5})\left(c_{2}-c_{3}\right)\right) & \\
z_{1}=0, z_{2}^{2}=\frac{1}{2}(7+3 \sqrt{5}) z_{3}^{2}, & & \approx \frac{8 \sqrt{5} \lambda^{2}}{45 c_{1}^{2}}\left(c_{2}-c_{3}\right)(\mathrm{x} 2), \\
3 & & -2 \lambda+\frac{\lambda^{2}}{45 c_{1}^{2}}\left(45\left(c_{2}+c_{3}\right)-11 \sqrt{5}\left(c_{2}-c_{3}\right)\right) \\
z_{3}^{2} \approx \frac{\lambda}{6 c_{1}}(3-\sqrt{5}) & & \approx \frac{8 \sqrt{5} \lambda^{2}}{45 c_{1}^{2}}\left(c_{2}-c_{3}\right)(\mathrm{x} 2), \\
4 & +\frac{\lambda^{2}}{540 c_{1}^{3}}(3-\sqrt{5})\left(45\left(c_{2}+c_{3}\right)-11 \sqrt{5}\left(c_{2}-c_{3}\right)\right) & & -2 \lambda+\frac{\lambda^{2}}{45 c_{1}^{2}}\left(45\left(c_{2}+c_{3}\right)-11 \sqrt{5}\left(c_{2}-c_{3}\right)\right) \\
z_{1}^{2}=z_{2}^{2}=z_{3}^{2}, & & \approx \frac{\lambda^{2}}{10 c_{1}^{2}}(5+\sqrt{5})\left(c_{2}-c_{3}\right), \\
z_{3}^{2} \approx \frac{\lambda}{3 c_{1}}+\frac{\lambda^{2}}{90 c_{1}^{2}}\left(45\left(c_{2}+c_{3}\right)-11 \sqrt{5}\left(c_{2}-c_{3}\right)\right) & & -\frac{\lambda^{2}}{10 c_{1}^{2}}(5-\sqrt{5})\left(c_{2}-c_{3}\right), \\
z_{1}^{2}=\frac{1}{2}(3-\sqrt{5}) z_{3}^{2}, z_{2}^{2}=\frac{1}{2}(3+\sqrt{5}) z_{3}^{2}, & -2 \lambda+\frac{\lambda^{2}}{5 c_{1}^{2}}\left(5\left(c_{2}+c_{3}\right)-\sqrt{5}\left(c_{2}-c_{3}\right)\right) \\
z_{3}^{2} \approx \frac{\lambda}{4 c_{1}}+\frac{\lambda^{2}}{40 c_{1}^{3}}\left(5\left(c_{2}+c_{3}\right)-\sqrt{5}\left(c_{2}-c_{3}\right)\right) &
\end{array}
$$

Table 5: Small- $\lambda$ approximations to the branches shown in Table 4 for irreps $2 \pm$. For irreps $3 \pm$ change the sign in front of $\sqrt{5}$ wherever it occurs.

Branch Representative equations Growth rate eigenvalues Isotropy subgroup (generators)

$$
\begin{array}{llll}
1 & z_{1}=\frac{\lambda}{3 c_{1}}, z_{2}=z_{3}=z_{4}=0 & -\lambda, \frac{5}{3} \lambda(\mathrm{x} 3) & \mathcal{T} \times \mathbb{Z}_{2}^{c}\left(g_{3}, g_{5}^{2} g_{2 d}, g_{c}\right) \\
1^{\prime} & z_{1}=z_{2}=z_{3}=z_{4}=-\frac{\lambda}{3 c_{1}} & -\lambda, \frac{5}{3} \lambda(\mathrm{x} 3) & \mathcal{T} \times \mathbb{Z}_{2}^{c}\left(g_{2}, g_{3 d}, g_{c}\right) \\
2 & z_{1}=z_{4}=\frac{\lambda}{c_{1}}, z_{2}=z_{3}=0 & 5 \lambda(\mathrm{x} 2),-\lambda,-5 \lambda & \mathcal{D}_{3} \times \mathbb{Z}_{2}^{c}\left(g_{2 d}, g_{3}, g_{c}\right) \\
2^{\prime} & z_{1}=z_{2}=z_{3}=-\frac{\lambda}{c_{1}}, z_{4}=0, & 5 \lambda(\mathrm{x} 2),-\lambda,-5 \lambda & \mathcal{D}_{3} \times \mathbb{Z}_{2}^{c}\left(g_{3 d}, g_{5}^{4} g_{2 d}, g_{c}\right)
\end{array}
$$

Table 6: Solution branches bifurcating at $\lambda=0$ for irrep $4+$. Solutions obtained by interchanging any of the $z_{i}$ are also permissible. 


$\begin{array}{llll}1 & z_{1}=z_{2}=z_{3}=z_{4}= & -2 \lambda\left(1+2 c_{3} / c_{2}\right)(\mathrm{x} 3) & \mathcal{T}\left(g_{2}, g_{3 d}\right) \\ & \pm \sqrt{-\lambda / c_{2}} & -2 \lambda & \\ 1^{\prime} & z_{4}= \pm \sqrt{-\lambda / c_{2}} & -2 \lambda\left(1+2 c_{3} / c_{2}\right)(\mathrm{x} 3) & \mathcal{T}\left(g_{3}, g_{5}^{3} g_{2 d}\right) \\ & z_{1}=z_{2}=z_{3}=0 & -2 \lambda & \\ z_{1}=0, z_{2}=z_{3}=z_{4}= & \lambda \frac{\left(c_{2}+2 c_{3}\right)}{\left(4 c_{2}+5 c_{3}\right)}(\mathrm{x} 2) & \mathcal{D}_{3}\left(g_{5} g_{2 d}, g_{5}^{2} g_{2} g_{5}\right) \\ \pm \sqrt{-\lambda /\left(4 c_{2}+5 c_{3}\right)} & 4 \lambda \frac{\left(c_{2}+2 c_{3}\right)}{\left(4 c_{2}+5 c_{3}\right)},-2 \lambda & \\ z_{1}=z_{4}= \pm \sqrt{-\lambda /\left(4 c_{2}+5 c_{3}\right)} & \lambda \frac{\left(c_{2}+2 c_{3}\right)}{\left(4 c_{2}+5 c_{3}\right)}(\mathrm{x} 2) & \mathcal{D}_{3}\left(g_{2 d}, g_{3}\right) \\ 2^{\prime} & 4 \lambda \frac{\left(c_{2}+2 c_{3}\right)}{\left(4 c_{2}+5 c_{3}\right)},-2 \lambda & \\ z_{2}=z_{3}=0 & -5 \lambda \frac{\left(c_{2}+2 c_{3}\right)}{\left(4 c_{2}+3 c_{3}\right)}(\mathrm{x} 2) & \mathcal{D}_{3}^{z}\left(g_{3}, g_{2 d} g_{c}\right) \\ z_{1}=-z_{4}= \pm \sqrt{-\lambda /\left(4 c_{2}+3 c_{3}\right)} & 4 \lambda \frac{\left(c_{2}+2 c_{3}\right)}{\left(4 c_{2}+3 c_{3}\right)},-2 \lambda & \\ z_{2}=z_{3}=0 & -5 \lambda \frac{\left(c_{2}+2 c_{3}\right)}{\left(4 c_{2}+3 c_{3}\right)}(\mathrm{x} 2) & \mathcal{D}_{3}^{z}\left(g_{5} g_{2} g_{5}^{2}, g_{5}^{3} g_{2} g_{5}^{3} g_{2 d} g_{c}\right) \\ 3^{\prime} & 4 \lambda \frac{\left(c_{2}+2 c_{3}\right)}{\left(4 c_{2}+3 c_{3}\right)},-2 \lambda & \\ & z_{1}=z_{2}=z_{3}=z_{4} / 2= & 10 \lambda \frac{\left(c_{2}+2 c_{3}\right)}{\left(13 c_{2}+16 c_{3}\right)}(\mathrm{x} 2) & \mathcal{D}_{2}^{z}\left(g_{2} g_{2 d}, g_{2} g_{c}\right) \\ & \pm \sqrt{-\lambda /\left(4 c_{2}+3 c_{3}\right)} & -2 \lambda \frac{\left(c_{2}+2 c_{3}\right)}{\left(13 c_{2}+16 c_{3}\right)},-2 \lambda & \\ z_{1}=z_{2}=-z_{3}=-z_{4}= & 10 \lambda \frac{\left(c_{2}+2 c_{3}\right)}{\left(13 c_{2}+16 c_{3}\right)}(\mathrm{x} 2) & \mathcal{D}_{2}^{z}\left(g_{5}^{4} g_{2} g_{5}^{4} g_{2 d}, g_{5}^{4} g_{2} g_{5} g_{c}\right) \\ \pm \sqrt{-\lambda /\left(13 c_{2}+16 c_{3}\right)} & -2 \lambda \frac{\left(c_{2}+2 c_{3}\right)}{\left(13 c_{2}+16 c_{3}\right)},-2 \lambda & \\ 4^{\prime} & \quad z_{1}=0, z_{2}=z_{3}=2 z_{4}= & \\ \pm 2 \sqrt{-\lambda /\left(13 c_{2}+16 c_{3}\right)} & & \end{array}$

Table 7: Solution branches bifurcating at $\lambda=0$ for irrep 4-. Solutions obtained by interchanging any of the $z_{i}$ are also permissible. 
$z_{3}=z_{4}=z_{5}=\lambda / c_{2}$

$-\lambda$

$\mathcal{D}_{3} \times \mathbb{Z}_{2}^{c}$

$z_{1}=z_{2}=0$

$\lambda\left(\frac{3}{2} \pm \frac{1}{2} \sqrt{13+\frac{36 c_{1}\left(c_{1}+c_{2}\right)}{c_{2}^{2}}}\right)(\mathrm{x} 2) \quad\left(g_{3}, g_{2 d}, g_{c}\right)$

1' $\quad z_{1}=z_{2}=z_{4}=-\lambda / c_{2}$

$-\lambda$

$\mathcal{D}_{3} \times \mathbb{Z}_{2}^{c}$

$z_{3}=z_{5}=0$

$\lambda\left(\frac{3}{2} \pm \frac{1}{2} \sqrt{13+\frac{36 c_{1}\left(c_{1}+c_{2}\right)}{c_{2}^{2}}}\right)(\mathrm{x} 2)$

$\left(g_{5}^{2} g_{2 d}, g_{3 d}, g_{c}\right)$

$2 \quad z_{1}=-\lambda /\left(2 c_{1}+c_{2}\right)$

$-\lambda$

$\mathcal{D}_{5} \times \mathbb{Z}_{2}^{c}$

$z_{2}=z_{3}=z_{4}=z_{5}=0$

$\lambda\left(\frac{3}{2} \pm \frac{\sqrt{5} c_{2}}{2\left(2 c_{1}+c_{2}\right)}\right)$

$(\mathrm{x} 2)$

$\left(g_{2 d}, g_{5}, g_{c}\right)$

2'

$z_{1}=z_{2}=z_{3}=z_{4}=z_{5}$

$-\lambda$

$\mathcal{D}_{5} \times \mathbb{Z}_{2}^{c}$

$=\lambda /\left(2 c_{1}+c_{2}\right)$

$\lambda\left(\frac{3}{2} \pm \frac{\sqrt{5} c_{2}}{2\left(2 c_{1}+c_{2}\right)}\right)(\mathrm{x} 2)$

$\left(g_{2} g_{5}, g_{2} g_{2 d}, g_{c}\right)$

3

$$
\begin{array}{ll}
z_{1}=z_{3}= & -\lambda, 3 \lambda \\
z_{4}\left(1+a+2 \cos \left(\frac{\theta}{3}\right) \sqrt{1+a+a^{2}}\right) & \lambda-2 c_{1} z_{1}+2\left(2 c_{1}+c_{2}\right) z_{4} \\
z_{4}=z_{5}=-\frac{\lambda}{c_{2}\left(1+a+a^{2}\right)\left(4 \cos ^{2}\left(\frac{\theta}{3}\right)-1\right)} & \lambda+2\left(2 c_{1}+c_{2}\right) z_{1}-2\left(c_{1}+c_{2}\right) z_{4} \\
z_{2}=0, a=\frac{c_{1}}{c_{2}}, \cos \theta=\frac{1+2 a}{2 \sqrt{1+a+a^{2}}} & \lambda-2\left(c_{1}+c_{2}\right) z_{1}-2 c_{1} z_{4}
\end{array}
$$$$
\mathcal{D}_{2} \times \mathbb{Z}_{2}^{c}
$$

(3 solutions)

Table 8: Representative solution branches bifurcating at $\lambda=0$ for irrep $5+$. 


$$
\begin{aligned}
& 1 \\
& z_{3}=z_{4}=z_{5}= \pm \sqrt{\frac{-\lambda}{2 c_{3}+c 4 / 2+2 c_{5}}} \\
& -2 \lambda \\
& \mathcal{D}_{3}\left(g_{3}, g_{2 d}\right) \\
& z_{1}=z_{2}=0 \\
& \text { 1' } \quad z_{1}=z_{2}=z_{4}= \pm \sqrt{\frac{-\lambda}{2 c_{3}+c 4 / 2+2 c_{5}}} \\
& \frac{\lambda\left(-11 c_{3}-5 c_{4}-2 c_{5} \pm i 3 \sqrt{3} c_{3}\right)}{4 c_{3}+c_{4}+4 c_{5}}(\mathrm{x} 2) \\
& z_{3}=z_{5}=0 \\
& -2 \lambda \\
& \mathcal{D}_{3}\left(g_{5}^{2} g_{2 d}, g_{3 d}\right) \\
& \frac{\lambda\left(-11 c_{3}-5 c_{4}-2 c_{5} \pm i 3 \sqrt{3} c_{3}\right)}{4 c_{3}+c_{4}+4 c_{5}}(\mathrm{x} 2) \\
& z_{1}= \pm \sqrt{\frac{-\lambda}{6 c_{3}+5 c_{4} / 2+2 c_{5}}} \\
& -2 \lambda \\
& \mathcal{D}_{5}\left(g_{2 d}, g_{5}\right) \\
& z_{2}=z_{3}=z_{4}=z_{5}=0 \\
& \frac{\lambda\left(11 c_{3}+5 c_{4}+2 c_{5} \pm \sqrt{5} c_{3}\right)}{12 c_{3}+5 c_{4}+4 c_{5}}(\mathrm{x} 2) \\
& -2 \lambda \\
& \mathcal{D}_{5}\left(g_{2} g_{5}, g_{2} g_{2 d}\right) \\
& = \pm \sqrt{\frac{-\lambda}{6 c_{3}+5 c_{4} / 2+2 c_{5}}} \\
& 3 \quad z_{1}=z_{3}=z_{4}=z_{5}=z_{2} / 2 \\
& = \pm \sqrt{\frac{-\lambda}{10 c_{3}+4 c_{4}+4 c_{5}}} \\
& \frac{\lambda\left(11 c_{3}+5 c_{4}+2 c_{5} \pm \sqrt{5} c_{3}\right)}{12 c_{3}+5 c_{4}+4 c_{5}}(\mathrm{x} 2) \\
& -2 \lambda, \frac{\lambda\left(8 c_{3}+5 c_{4}+2 c_{5}\right)}{10 c_{3}+4 c_{4}+4 c_{5}} \\
& \frac{\lambda\left(14 c_{3}+5 c_{4}+2 c_{5}\right)}{10 c_{3}+4 c_{4}+4 c_{5}} \\
& \pm \frac{\lambda \sqrt{\left(14 c_{3}+5 c_{4}+2 c_{5}\right)\left(8 c_{3}+5 c_{4}+2 c_{5}\right)}}{10 c_{3}+4 c_{4}+4 c_{5}} \\
& \text { 3' } \quad z_{1}=-z_{3}= \\
& = \pm \sqrt{\frac{-\lambda}{10 c_{3}+4 c_{4}+4 c_{5}}} \\
& z_{2}=z_{4}=z_{5}=0 \\
& 4 \\
& z_{1}=-z_{3}= \pm \sqrt{-\left(8 c_{3}+5 c_{4}+2 c_{5}\right) \lambda / \xi} \\
& -2 \lambda, \frac{\lambda\left(8 c_{3}+5 c_{4}+2 c_{5}\right)}{10 c_{3}+4 c_{4}+4 c_{5}} \\
& \lambda\left(14 c_{3}+5 c_{4}+2 c_{5}\right) \\
& 10 c_{3}+4 c_{4}+4 c_{5} \\
& \pm \frac{\lambda \sqrt{\left(14 c_{3}+5 c_{4}+2 c_{5}\right)\left(8 c_{3}+5 c_{4}+2 c_{5}\right)}}{10 c_{3}+4 c_{4}+4 c_{5}} \\
& \frac{\lambda\left(\left(11 c_{3}+5 c_{4}+2 c_{5}\right)^{2}+27 c_{3}^{2}\right)}{\xi} \\
& z_{4}=-z_{5}= \pm \sqrt{-\left(14 c_{3}+5 c_{4}+2 c_{5}\right) \lambda / \xi} \quad-\frac{2 \lambda\left(14 c_{3}+5 c_{4}+2 c_{5}\right)\left(8 c_{3}+5 c_{4}+2 c_{5}\right)}{\xi}(\mathrm{x} 2) \\
& z_{2}=0 \quad-2 \lambda, 0 \\
& \xi=108 c_{3}^{2}+78 c_{3} c_{4}+84 c_{3} c_{5}+15 c_{4}^{2}+36 c_{4} c_{5}+12 c_{5}^{2} \\
& 4 \\
& z_{1}=z_{3}=z_{2} / 2=\left(z_{4}+z_{5}\right) / 2=\quad \frac{\lambda\left(\left(11 c_{3}+5 c_{4}+2 c_{5}\right)^{2}+27 c_{3}^{2}\right)}{\xi} \\
& \pm \sqrt{-\left(14 c_{3}+5 c_{4}+2 c_{5}\right) \lambda / \xi} \\
& -\frac{2 \lambda\left(14 c_{3}+5 c_{4}+2 c_{5}\right)\left(8 c_{3}+5 c_{4}+2 c_{5}\right)}{\xi}(\mathrm{x} 2) \\
& \left(z_{4}-z_{5}\right) / 2=\quad-2 \lambda, 0 \\
& \pm \sqrt{-\left(8 c_{3}+5 c_{4}+2 c_{5}\right) \lambda / \xi} \\
& \mathbb{Z}_{2}^{\text {refl }}\left(g_{2} g_{2 d} g_{c}\right) \\
& \mathbb{Z}_{2}^{\text {refl }}\left(g_{2 d} g_{c}\right)
\end{aligned}
$$

Table 9: Representative solution branches bifurcating at $\lambda=0$ for irrep $5-$. 


Irrep Axial (maximal) isotropy subgroups

$\begin{array}{ll}2 \text { and } 3 & \mathbb{Z}_{2}, \mathbb{Z}_{3}, \mathbb{Z}_{5} \\ 4 & \mathcal{T}, \mathcal{D}_{3} \\ 5 & \mathcal{D}_{3}, \mathcal{D}_{5},\left(\mathcal{D}_{2}\right)\end{array}$

Table 10: Axial isotropy subgroups for each nontrivial irrep of $\mathcal{I}$. Maximal, but non-axial, isotropy subgroups are given in brackets. 